\title{
The Impact of Aging on
} \section{Macroautophagy in the Pre-ovulatory Mouse Oocyte}

\author{
Alexandra E. Peters ${ }^{1,2 *}$, Shandelle J. Caban ${ }^{1,2}$, Eileen A. McLaughlin ${ }^{1,2,3,4}$, \\ Shaun D. Roman ${ }^{1,2,5}$, Elizabeth G. Bromfield ${ }^{1,2,6}$, Brett Nixon ${ }^{1,2}$ and \\ Jessie M. Sutherland ${ }^{1,2}$
}

1 Priority Research Centre for Reproductive Science, Schools of Biomedical Science \& Pharmacy and Environmental \& Life Sciences, University of Newcastle, Callaghan, NSW, Australia, ${ }^{2}$ Pregnancy and Reproduction Program, Hunter Medical Research Institute, New Lambton Heights, NSW, Australia, ${ }^{3}$ School of Science, Western Sydney University, Penrith, NSW, Australia, ${ }^{4}$ School of Biological Sciences, Faculty of Science, The University of Auckland, Auckland, New Zealand, ${ }^{5}$ Priority Research Centre for Drug Development, The University of Newcastle, Callaghan, NSW, Australia, ${ }^{6}$ Department of Biochemistry and Cell Biology, Faculty of Veterinary Medicine, Utrecht University, Utrecht, Netherlands

\section{OPEN ACCESS}

Edited by:

Karin Lykke-Hartmann, Aarhus University, Denmark

Reviewed by:

Shail K. Chaube,

Banaras Hindu University, India

Zhao-Jia Ge,

Qingdao Agricultural University, China

*Correspondence:

Alexandra E. Peters

alexandra.peters@uon.edu.au; c3232460@uon.edu.au

Specialty section:

This article was submitted to Cell Growth and Division,

a section of the journal

Frontiers in Cell and Developmental

Biology

Received: 07 April 2021 Accepted: 04 June 2021

Published: 29 June 2021

Citation:

Peters AE, Caban SJ, McLaughlin EA, Roman SD,

Bromfield EG, Nixon $B$ and Sutherland JM (2021) The Impact of Aging on Macroautophagy in the Pre-ovulatory Mouse Oocyte. Front. Cell Dev. Biol. 9:691826. doi: 10.3389/fcell.2021.691826
Accompanying the precipitous age-related decline in human female fertility is an increase in the proportion of poor-quality oocytes within the ovary. The macroautophagy pathway, an essential protein degradation mechanism responsible for maintaining cell health, has not yet been thoroughly investigated in this phenomenon. The aim of this study was to characterize the macroautophagy pathway in an established mouse model of oocyte aging using in-depth image analysis-based methods and to determine mechanisms that account for the observed changes. Three autophagy pathway markers were selected for assessment of gene and protein expression in this model: Beclin 1; an initiator of autophagosome formation, Microtubule-associated protein 1 light chain 3B; a constituent of the autophagosome membrane, and lysosomal-associated membrane protein 1; a constituent of the lysosome membrane. Through quantitative image analysis of immunolabeled oocytes, this study revealed impairment of the macroautophagy pathway in the aged oocyte with an attenuation of both autophagosome and lysosome number. Additionally, an accumulation of amphisomes greater than $10 \mu \mathrm{m}^{2}$ in area were observed in aging oocytes, and this accumulation was mimicked in oocytes treated with lysosomal inhibitor chloroquine. Overall, these findings implicate lysosomal dysfunction as a prominent mechanism by which these age-related changes may occur and highlight the importance of macroautophagy in maintaining mouse pre-ovulatory oocyte quality. This provides a basis for further investigation of dysfunctional autophagy in poor oocyte quality and for the development of therapeutic or preventative strategies to aid in the maintenance of pre-ovulatory oocyte health.

Keywords: autophagy, amphisome, lysosome, autophagosome, reproductive system, protein degradation, oocyte quality

Abbreviations: Becn1/BECN1, Beclin-1; DAP1, death associated protein 1; EEA1, early endosome antigen 1; GAPDH, anti-glyceraldehyde 3-phosphate dehydrogenase; KIF: kinesin super family protein; Lamp1/LAMP1, lysosomal associated membrane protein 1; LC3B, microtubule associated protein 1 light chain 3B; Map 1lc3/L3c, microtubule associated protein 1 light chain 3; mTOR, mammalian target of rapamycin; PI3K, phosphoinositide 3-kinase; Ppia, peptidylprolyl isomerase A; RAB, Ras-associated binding protein; STIM1, stromal interaction molecule 1; TFEB, transcription factor EB; ULK, unc-51-like kinase 1. 


\section{INTRODUCTION}

Infertility is a major health burden affecting an estimated $15 \%$ of couples globally, with a female factor implicated in approximately half of all cases (Vayena et al., 2002; Agarwal et al., 2005; Vander Borght and Wyns, 2018). Notably, female fertility decreases significantly after the age of 35 (Spira, 1988; te Velde and Pearson, 2002; Leridon, 2004), creating a restricted fertility window that is dictated by the number and quality of oocytes within the ovarian reserve (te Velde and Pearson, 2002; Doherty and Pal, 2011). During mammalian embryonic development, all oocytes enter meiosis and arrest in prophase I until selected for ovulation some decades later (Hsueh et al., 2015). This prolonged stage of cell cycle arrest renders the oocyte vulnerable to damage from a variety of insults such as the oxidative stress and inflammation associated with folliculogenesis and ovulation (Chao et al., 2005; Goud et al., 2008; Miyamoto et al., 2010; Lliberos et al., 2021). Chronic exposure to these factors throughout a female's reproductive life can cause damage to the intracellular environment of the oocyte and its immediate supportive cells, contributing to reduced oocyte quality. Evidence of this wellestablished phenomenon is apparent in the elevated aneuploidy rates of oocytes retrieved from women ${ }^{1}$ over 35 ; where at least $50 \%$ of the oocytes in women aged $>40$ are rendered non-viable (Kuliev et al., 2003; De Bruin and Te Velde, 2004; Fu et al., 2014). Cumulatively, the limiting nature of female fertility results in age-related infertility being the single largest cause of human infertility (Doherty and Pal, 2011; Agarwal et al., 2015). Despite various pathways' involvement in oocyte aging, there are minimal therapeutic or preventative treatments available to maintain or improve oocyte quality or enhance the success of interventions such as assisted reproductive technologies (Newman et al., 2019; Peters et al., 2020). This situation provides a clear imperative for further investigation into the molecular pathways that underpin female age-related infertility.

One such pathway that warrants consideration in the context of oocyte aging is autophagy; a ubiquitous protein degradation pathway that plays a critical role in cellular protein homeostasis (proteostasis). Autophagy pathways can be activated in response to a diverse range of stressors such as oxidative stress, hypoxia, thermal fluctuations, and nutrient deprivation (Levine and Kroemer, 2008). All autophagy pathways utilize the lysosome, an acidic intracellular vesicle containing hydrolytic enzymes to facilitate the breakdown of aged, damaged, and dysfunctional proteins and organelles (Feng et al., 2014; Klionsky et al., 2016). Importantly, somatic cell studies have demonstrated that the efficiency of autophagy pathways decreases during aging, particularly in long-lived cells such as neurons and cardiac myocytes. This decreased efficiency results from attenuation of lysosomal degradation capacity combined with reduced production, fusion, and elimination of autophagosomes (Terman, 1995; Terman and Brunk, 2004; Essick and Sam, 2010).

\footnotetext{
${ }^{1}$ The use of the term 'women' here refers to people assigned female at birth and possessing a female reproductive system. The authors acknowledge that not all people who have female reproductive systems are women.
}

Captured under the umbrella of autophagy are numerous degradation pathways responsible for targeting different cellular components. However, the autophagy pathway with the most diverse and best-characterized roles is macroautophagy (Klionsky and Codogno, 2013). The macroautophagy pathway employs the autophagosome, a double membraned intracellular vesicle to sequester and deliver cargo to the lysosome (Seglen et al., 1990; Feng et al., 2014) (Figure 1). This cargo, usually consisting of aged or damaged proteins, is destined for hydrolysis into its core amino acid building blocks, which are then recycled for use within cellular biosynthetic pathways (Seglen et al., 1990; Feng et al., 2014). Among the key macroautophagy protein components are Beclin-1 (BECN1; involved in the promotion of autophagosome formation), microtubule associated protein 1 light chain 3B (LC3B; an essential constituent of the autophagosome membrane), and lysosomal associated membrane protein 1 (LAMP1; a primary constituent of the lysosome membrane) (Feng et al., 2014; Klionsky et al., 2016) (Figure 1).

Within the ovarian environment, there is evidence that macroautophagy is essential for promoting oocyte survival and development. By way of illustration, the knockdown of Becn 1 in porcine oocytes leads to a reduction in both BECN1 and LC3B protein expression and promotes a suite of phenotypic responses. These responses include reduced polar body extrusion during meiosis, increased DNA damage, and impaired mitochondrial function; changes that ultimately compromise the developmental potential of the oocyte (Shen et al., 2018). Comparable effects have also been documented in response to the use of an upstream inhibitor of autophagy, LY294002 (Shen et al., 2018). Furthermore, the selective knockout of Becnl or autophagyrelated gene, Atg7, significantly reduced or eliminated the oocyte pool within the ovaries of the Becn 1 and Atg7 deficient mice, respectively (Gawriluk et al., 2011). It should be noted that autophagy can fulfill dual roles in the ovary, whereby it is not only responsible for promoting oocyte survival but alternatively, can promote cell death. Indeed, autophagy pathways operating alongside other cell death mechanisms, such as apoptosis and necroptosis, have been implicated in follicular atresia, an important mechanism responsible for oocyte loss with increasing age (Escobar et al., 2008; Yadav et al., 2018; Chaudhary et al., 2019).

In addition to promoting oocyte survival and growth, autophagy activation has been detected in response to various stress-inducing stimuli. For instance, elevated levels of GFPLC3 puncta, indicative of autophagy pathway activation, have been detected in mouse oocytes subjected to cold stress (Bang et al., 2014). Activation of autophagy has also been observed in bovine oocytes in response to heat shock (Latorraca et al., 2020). Similarly, the ovulated oocytes of obese mice present with increased levels of autophagy accompanied by mitochondrial dysfunction compared to those harvested from lean mice ( $\mathrm{Wu}$ et al., 2015). Finally, exposure of female mice to the dietary toxicant acrylamide has been shown to increase the expression of autophagy-related genes such as Lc3 (a biomarker of autophagy activation) within the ovaries of treated animals (Aldawood et al., 2020). Taken together, such studies allude 


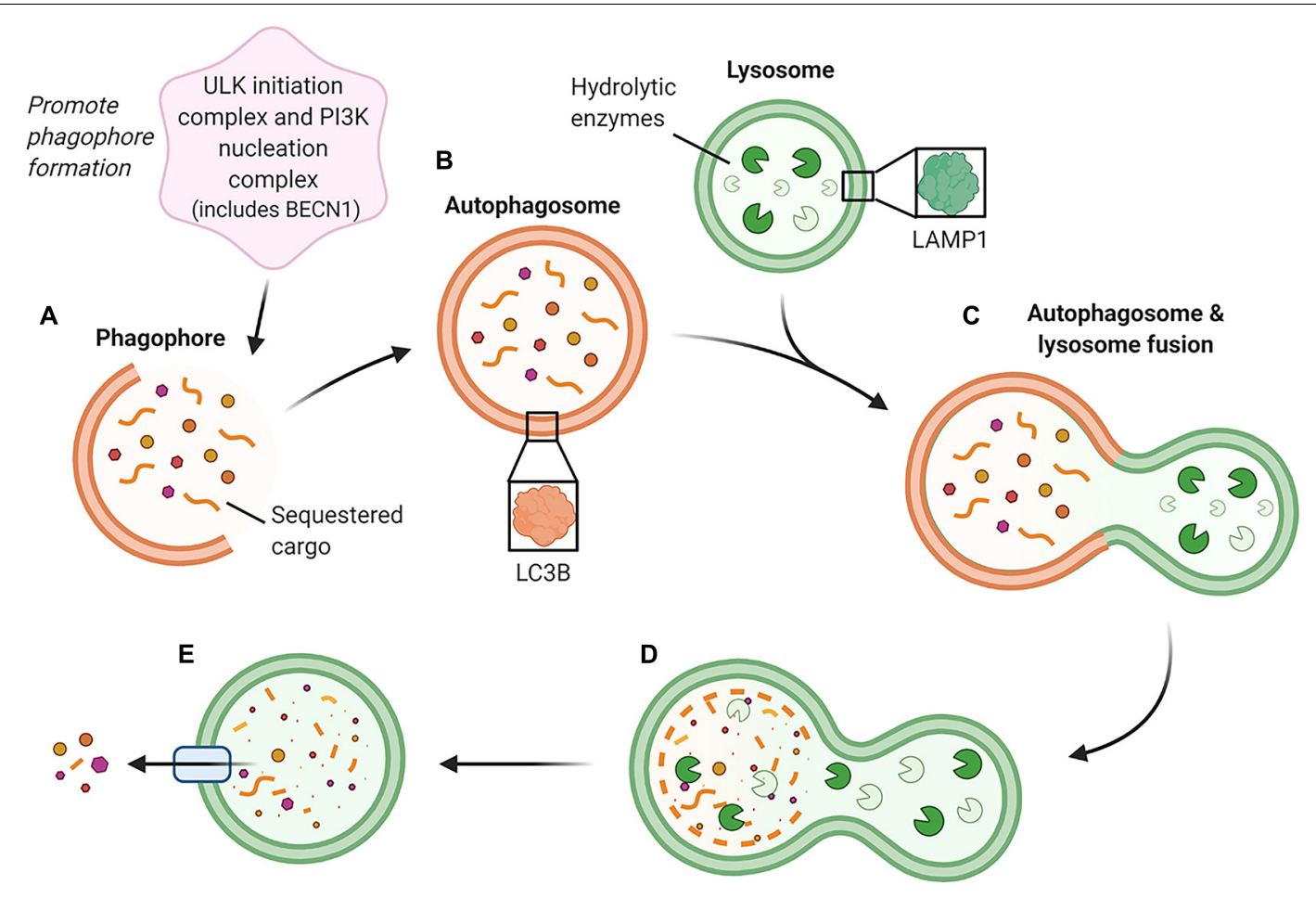

Degradation \& recycling

Autolysosome

FIGURE 1 | Schematic of the macroautophagy pathway. This pathway is initiated by two groups of proteins, the ULK (unc-51-like kinase 1) initiation complex and the PI3K (phosphoinositide 3-kinase) nucleation complex containing BECN1, which generate the phagophore (immature autophagosome) (A). As the phagophore continues to grow and sequester cargo, it forms a completed double-membraned vesicle termed the autophagosome, which encompasses the cargo to be degraded (B). The autophagosome then proceeds to fuse with an acidic lysosome containing hydrolytic enzymes (C) to form an autolysosome. (D) Within the autolysosome, the encapsulated cargo is broken down into its basic building blocks and is then extruded back into the cell cytoplasm for recycling (E). (Feng et al., 2014; Klionsky et al., 2016) Created with BioRender.com.

to the demonstrable importance of autophagy in maintaining oocyte health. However, the basis by which autophagy operates on a subcellular level in the oocyte and its alteration during reproductive aging remains largely unexplored. To address this knowledge gap, this study aimed to characterize components of the macroautophagy pathway in preovulatory oocytes and assess how the fidelity of this pathway is impacted during natural maternal aging.

\section{MATERIALS AND METHODS}

\section{Animal Ethics}

Research animals in this study were handled, monitored, and euthanized in accordance with NSW Animal Research Act 1998, NSW Animal Research Regulation 2010 and the Australian Code for the Care and Use of Animals for Scientific Purposes 8th Ed. as approved by the University of Newcastle Animal Care and Ethics Committee (approval number A-2018-803). C57/BL6 $\times$ CBA hybrid (F1) mice were bred by the University of Newcastle Animal Services Unit and housed under a $12 \mathrm{~h}$ light/12 $\mathrm{h}$ dark cycle at a constant temperature of $21-22^{\circ} \mathrm{C}$ with food and water supplied ad libitum. Mice were euthanized immediately before oocyte collection via carbon dioxide inhalation, and death was confirmed through subsequent cervical dislocation.

C57/BL6 $\times$ CBA hybrid (F1) mice were utilized at two critical time points: 4-6 weeks old, equivalent to a human with initial fertility, and 12-14 months old, equivalent to a human with declining fertility. These time points were selected based on an established model of oocyte aging in our research group that exhibits similar age-related changes in oocyte quality and quantity as humans (Camlin et al., 2017b).

\section{Oocyte Collection}

Unstimulated ovaries were removed from euthanized mice aged between 4 and 6 weeks (young time point) or 12-14 months of age (aged time point). Ovaries were dissociated by repeatedly puncturing pre-ovulatory follicles with a 27-gauge needle to release mature pre-ovulatory oocytes as cumulus-oocyte complexes into pre-warmed $\left(37^{\circ} \mathrm{C}\right)$ M2 media (Merck, Darmstadt, Germany) supplemented with $2.5 \mu \mathrm{M}$ milrinone to maintain prophase I arrest. Media droplets were kept under embryo tested mineral oil (Merck) in $35 \mathrm{~mm}$ Petri dishes 
(Cellstar, Baltimore, MD, United States). Cumulus cells were mechanically removed via repeated aspiration with a narrow pipette and washed through M2 media droplets until no cumulus cells or debris remained.

\section{RNA Extraction, Reverse Transcription, and Quantitative PCR (RT-qPCR)}

Total RNA was extracted from 40 oocytes per replicate following removal of the zona pellucida via incubation in acid Tyrode's solution (Merck). RNA was subsequently snap-frozen in liquid nitrogen and stored at $-80^{\circ} \mathrm{C}$. Reverse transcription was performed to generate cDNA using a TaqMan Gene Expression Cells-to-CT kit (Thermo Fisher Scientific, Waltham, MA, United States) as per the manufacturer's instructions and stored at $-80^{\circ} \mathrm{C}$.

Quantitative real-time PCR (RT-qPCR) was performed on cDNA using TaqMan assay probes and TaqMan gene expression master mix (Thermo Fisher Scientific) according to the manufacturer's instructions. The assay probes used were Becn1 (assay ID. Hs01007018_m1), Map1lc3 (assay ID. Mm00782868_sH), and Lamp1 (assay ID. Mm00495262_m1). RT-qPCR for each biological replicate was performed in triplicate using $2 \mu \mathrm{l}$ of cDNA for 40 amplification cycles $\left(50^{\circ} \mathrm{C}\right.$ preincubation for $120 \mathrm{~s}, 95^{\circ} \mathrm{C}$ pre-incubation for $600 \mathrm{~s}$, followed by 40 cycles of $95^{\circ} \mathrm{C}$ for $15 \mathrm{~s}$ and $60^{\circ} \mathrm{C}$ for $60 \mathrm{~s}$ ) with a LightCycler 96 (Roche Diagnostics, Basel, Switzerland). For each sample, a replicate omitting the reverse transcriptase enzyme was used as a negative control. All data were analyzed using LightCycler 96 software (Version 1.1) according to the equations of Schmittgen and Livak (2008). Data were normalized to the housekeeping gene, peptidylprolyl isomerase A (Ppia) (assay ID. Mm02342429_g1), and then presented relative to young oocytes where appropriate. Ppia was chosen as an appropriate control due to its previous use in oocytes, its maintained expression levels between groups, and detection at earlier cycle values than that of the genes of interest (Mihalas et al., 2019).

\section{SDS-PAGE and Immunoblotting}

Protein was extracted from oocytes by incubating in NuPAGE $4 \times$ lithium dodecyl sulfate (LDS) sample buffer (Invitrogen, Waltham, MA, United States) diluted in RIPA buffer $(150 \mathrm{mM}$ $\mathrm{NaCl}, 50 \mathrm{mM}$ Tris $\mathrm{pH} 8,0.1 \% \mathrm{w} / \mathrm{v}$ SDS, $0.5 \% \mathrm{w} / \mathrm{v}$ sodium deoxycholate, $1 \% \mathrm{v} / \mathrm{v}$ Triton $\mathrm{X}$-100) supplemented with protease/phosphatase inhibitor cocktail (Thermo Fisher Scientific) and $4 \% \mathrm{v} / \mathrm{v} \beta$-mercaptoethanol (Merck) at $100^{\circ} \mathrm{C}$ for $10 \mathrm{~min}$. Protein extracts equivalent to 50 oocytes per lane were loaded into a $12 \%$ Bis-Tris Bolt pre-cast gel (Invitrogen), and electrophoresis was performed at a constant voltage of $130 \mathrm{~V}$. This was followed by western transfer onto a $0.45 \mu \mathrm{m}$ PVDF membrane (GE Healthcare, Amersham, Buckinghamshire, United Kingdom) at a constant current of $350 \mathrm{~mA}$ for $90 \mathrm{~min}$. Membranes were then blocked with $5 \%$ w/v skim milk diluted in Tris-buffered saline (TBS) with 1\% v/v Tween-20 (TBST) for $2 \mathrm{~h}$ at room temperature. Following blocking, membranes were incubated with primary antibodies specific for BECN1,
LC3B, or LAMP1 diluted as in Supplementary Table 1 in $1 \% \mathrm{w} / \mathrm{v}$ skim milk-TBST at $4^{\circ} \mathrm{C}$ overnight. Membranes were washed 3 times $(2 \times 10 \mathrm{~min}$ followed by $1 \times 30 \mathrm{~min})$ in TBST and then incubated with horseradish peroxidase-conjugated secondary antibody diluted in $1 \% \mathrm{w} / \mathrm{v}$ skim milk-TBST for $2 \mathrm{~h}$ at room temperature. Blots were washed 3 times $(2 \times 10 \mathrm{~min}$ followed by $1 \times 30 \mathrm{~min})$ again in TBST prior to being developed using an enhanced chemiluminescence kit (GE Healthcare). After development, membranes were stripped using Western ReProbe (G-Biosciences, Maryland Heights, $\mathrm{MO}$, United States) and re-probed with anti-glyceraldehyde 3-phosphate dehydrogenase (GAPDH) antibodies as in Supplementary Table 1 in TBST. Densitometry was performed using Amersham Imager 600 software (GE Healthcare), and protein expression of target proteins was normalized against GAPDH loading control.

\section{Immunocytochemistry}

Following oocyte collection, live cells were rinsed 3 times in phosphate-buffered saline (PBS) containing $3 \mathrm{mg} / \mathrm{ml}$ polyvinylpyrrolidone (PVP). They were then fixed and permeabilized in $2 \% \mathrm{v} / \mathrm{v}$ paraformaldehyde containing $0.5 \%$ $\mathrm{v} / \mathrm{v}$ Triton-X for $30 \mathrm{~min}$. Fixed oocytes were rinsed 3 times again in PBS/PVP before being blocked with $7 \% \mathrm{v} / \mathrm{v}$ normal goat serum (Merck) in $1 \% \mathrm{w} / \mathrm{v}$ BSA with $0.1 \% \mathrm{v} / \mathrm{v}$ Tween-20 in PBS (PBST) for $1 \mathrm{~h}$ at room temperature. The oocytes were then incubated with primary antibodies specific for BECN1, LC3B, LAMP1, or EEA1 diluted as in Supplementary Table 1 in $1 \% \mathrm{w} / \mathrm{v}$ BSA-PBST overnight at $4^{\circ} \mathrm{C}$. Oocytes were washed 3 times $(2 \times 5 \mathrm{~min}$ followed by $1 \times 60 \mathrm{~min})$ in $1 \% \mathrm{w} / \mathrm{v}$ BSA-PBST before being incubated with relevant Alexa Fluor-conjugated secondary antibodies (Thermo Fisher Scientific) diluted 1:500 in $1 \% \mathrm{w} / \mathrm{v}$ BSA-PBST for $1 \mathrm{~h}$ at room temperature. All experiments included secondary antibody only controls in which the primary antibody was substituted with antibody/wash buffer (Supplementary Figure 1). Oocytes were once again washed three times $(2 \times 5 \mathrm{~min}$ followed by $1 \times 60 \mathrm{~min})$ in $1 \% \mathrm{w} / \mathrm{v}$ BSAPBST and counterstained with 4', 6-diamidino-2-phenylindole (DAPI) diluted 1:5000 in PBS for $15 \mathrm{~min}$ at room temperature. Oocytes were mounted onto 12 well slides (Thermo Fisher Scientific) in $1 \mu \mathrm{L}$ of Citifluor Glycerol Solution AF2 (Citifluor Ltd., London, United Kingdom) per well. Pre-ovulatory oocytes with a chromatin formation indicative of reduced capacity to complete embryonic development (non-surrounded nucleus) (Christians et al., 1999) were excluded from each group upon assessing the chromatin arrangement stained with DAPI.

\section{Confocal Imaging}

Oocyte images were captured via confocal microscopy on an Olympus FV1000 confocal microscope under a $60 \times$ oil immersion lens. For each oocyte, a $z$-stack was performed with $1 \mu \mathrm{m}$ intervals for $15 \mu \mathrm{m}$ total through the center of the oocyte capturing the whole nucleolus. Different color channels were imaged sequentially to avoid bleed-through, and a Kalman filter was applied to reduce background fluorescence. Excitation lasers used were $405 \mathrm{~nm}$ (DAPI), 559 nm (Alexa Fluor 555), and 635 nm 
(Alexa Fluor 633) with the emission imaged for analysis. In each group, 5-15 mature oocytes were imaged for analysis.

\section{Puncta Analysis: Measurement of Puncta Per Cell}

Firstly, slices of each $15 \mu \mathrm{m} z$-stack were overlaid into a single image for puncta analysis using Fiji software (extension of ImageJ version 1.53c) (Schindelin et al., 2012). For each image, the unit of measurement was converted from pixels to microns using the scale bar, followed by converting the image to an 8-bit format. The MorphoLibJ plugin (Legland et al., 2016) was then used, with the morphological filter 'White Top Hat' to reduce background fluorescence that can obscure puncta size (Qvarnström et al., 2004). Next, the images underwent thresholding to isolate the puncta visible in the original image (Figure 2). Thresholds were kept consistent across experimental groups within each replicate. Finally, the number of puncta was assessed, producing a numbered list of each puncta and its associated area in $\mu \mathrm{m}^{2}$. For each oocyte, the output for the number of puncta was sorted by size in Microsoft Excel, and the total size distribution was compared between groups in GraphPad Prism 8.4.3 (San Diego, CA, United States). Size categories assessed were defined by the relevant vesicle labeled by each marker along with additional size categories for other puncta detected to assess all potential differences within each dataset. These size categories included the recorded autophagosome size (Mizushima et al., 2002) $\left(0.5-1.5 \mu \mathrm{m}\right.$ in diameter or $0.196-1.767 \mu \mathrm{m}^{2}$ in area) for LC3B and recorded lysosome size (Ponsford et al., 2020; Trivedi et al., 2020) $\left(0.03-0.5 \mu \mathrm{m}^{2}\right.$ in area) for LAMP1. Additional puncta detected outside of these categories were assessed in arbitrary categories increasing by $1 \mu \mathrm{m}^{2}$ up to $>10 \mu \mathrm{m}^{2}$ for LC3B and BECN1, and increasing by $0.1 \mu \mathrm{m}^{2}$ up to $>1 \mu \mathrm{m}^{2}$
A

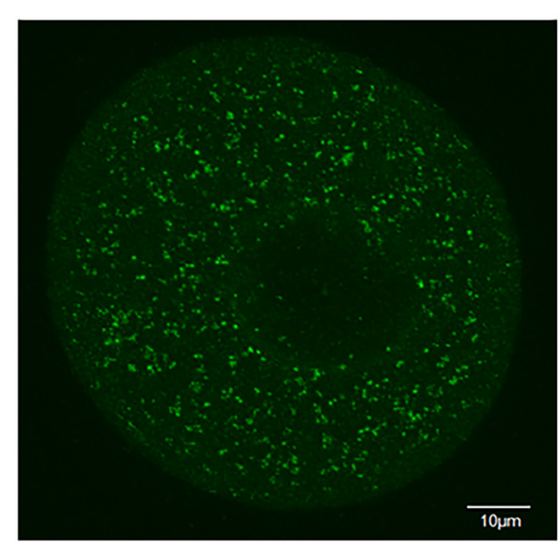

D

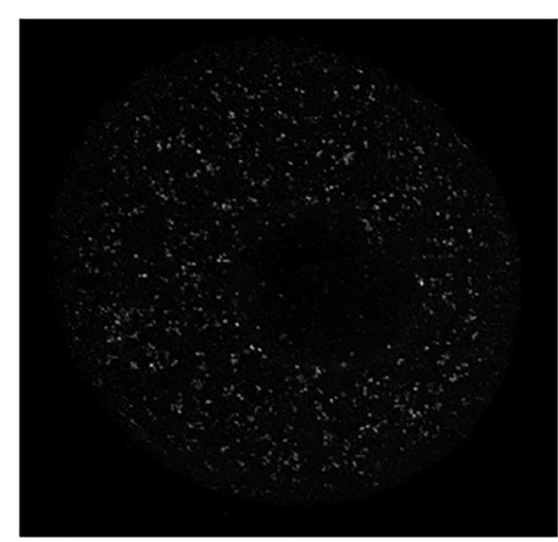

B

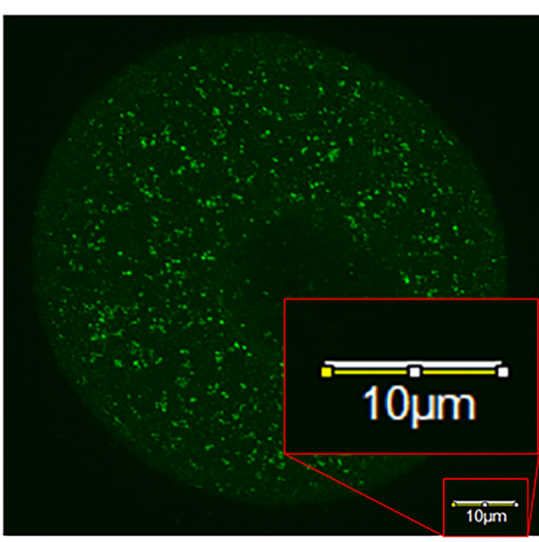

E

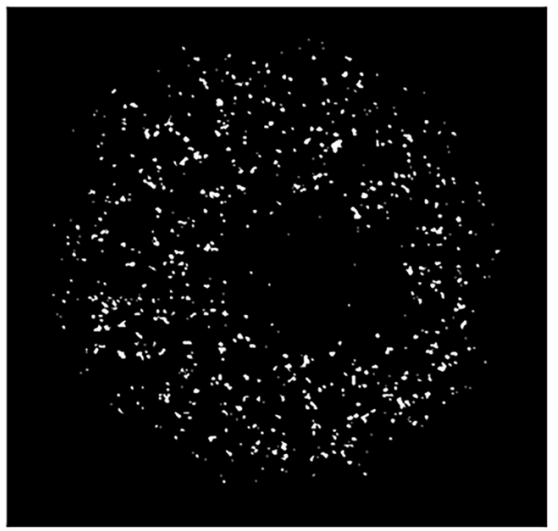

C

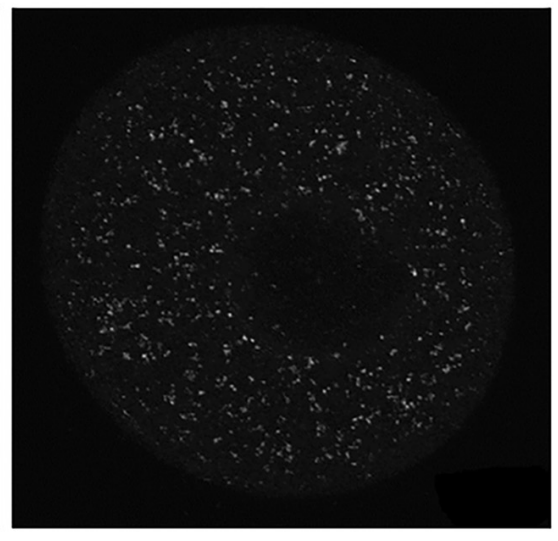

F

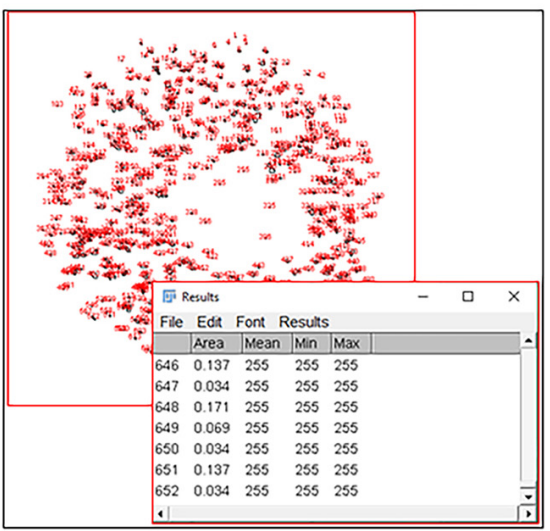

FIGURE 2 | Details of the puncta analysis method utilized to analyze puncta size distribution within immunolabeled oocyte images. (A) Firstly, the single-channel image of antibody staining to be analyzed with a scale bar of known size was acquired. (B) The scale bar was traced using the straight-line tool, and the scale of the image was adjusted from pixels to microns (Analyze $>$ Set Scale > Input scale bar size as known distance). The scale bar was then circled and deleted using an area selection tool to remove from the image. (C) The image was converted to an 8-bit grayscale format for further analysis (Image $>$ Type $>$ 8-bit). (D) White top hat transformation (Plugins > Morpholibj > Morphological filters) was then performed. To do this, the 'White top hat' operation was selected, and parameters were adjusted to obtain a reduced background and enhance features of interest. (E) The image was then thresholded (Image $>$ Adjust $>$ Threshold) by selecting the threshold that most accurately represented the detail of the initial image ( $T=20$ here). (F) Finally, the 'analyze particles' feature was selected (Analyze $>$ Analyze particles) to produce a dialog box containing the number and size of each puncta measured. This information was copied into Excel, where puncta were sorted by size and categorized as desired. 
for LAMP1. The differences between experimental groups for each category were then determined through statistical analysis as detailed below.

\section{Puncta Analysis: Periphery Measurements}

The proportion of $\mathrm{LC} 3 \mathrm{~B}$ and BECN1 puncta greater than $10 \mu \mathrm{m}^{2}$ residing at the cell's periphery was also measured. This measurement was carried out using the same process as above (puncta analysis: measurement of puncta per cell) with an extra step to select for the outer $10 \mu \mathrm{m}$ of the cell. The number of puncta greater than $10 \mu \mathrm{m}^{2}$ in the periphery of each cell was then compared between young and aged groups, and differences were determined via statistical analysis as detailed below.

\section{Colocalization Analysis}

Using Fiji software (an extension of ImageJ version 1.53c), each two-channel image was split into its individual channels, and the morphological top-hat filter was applied (MorphoLibJ plugin). The Coloc 2 plugin was then used to produce an output displaying several statistical colocalization tests showing the relationship between the localization of the two different channels. From these data, Pearson's correlation coefficients (no threshold) were obtained as indicators of colocalization of the target proteins within each oocyte, and groups were then compared.

\section{Proximity Ligation Assay}

Proximity ligation assays (PLA) were performed on fixed oocytes using a Duolink In Situ Red Starter kit (Merck) as per the manufacturer's instructions with slight modification. Briefly, oocytes were blocked with Duolink blocking solution for $1 \mathrm{~h}$ at $37^{\circ} \mathrm{C}$ and then incubated with primary antibodies LC3B and EEA1 diluted as in Supplementary Table 1 in Duolink antibody buffer overnight at $4^{\circ} \mathrm{C}$. Labeled oocytes were then washed with PBS/PVP supplemented with $1 \% \mathrm{v} / \mathrm{v}$ Tween$20(3 \times 10 \mathrm{~min})$ before incubation with oligonucleotideconjugated secondary antibodies (positive and negative PLA probes) for $1 \mathrm{~h}$ at $37^{\circ} \mathrm{C}$. After additional washes $(3 \times 10 \mathrm{~min})$, the ligation and amplification of PLA probes were conducted according to the manufacturer's instructions. Finally, oocytes were counterstained with DAPI at a 1:5,000 dilution in PBS for $15 \mathrm{~min}$ at room temperature. Positive control incubations included antibody pairs targeting proteins expected to interact, including $\alpha$-tubulin and $\beta$-tubulin (Supplementary Table 1). Negative control incubations used to confirm the specificity of the assay included antibody pairs targeting proteins that would not be expected to interact; LC3B and PIWIL1 (Supplementary Table 1), as well as the omission of primary antibodies (Supplementary Figure 2).

\section{Chloroquine Treatment}

Titration of effective doses of chloroquine phosphate (Abcam) was first performed using working solutions of between 0 and $400 \mu \mathrm{M}$ chloroquine prepared in M2 media (Merck) supplemented with $2.5 \mu \mathrm{M}$ milrinone to maintain prophase
I arrest. Live oocytes were washed through $2 \times 100 \mu \mathrm{L}$ droplets of each relevant treatment before incubation in a third $100 \mu \mathrm{L}$ droplet under mineral oil (Merck) in a $35 \mathrm{~mm}$ embryo tested Petri dish (Cellstar) for $6 \mathrm{~h}$ at $37^{\circ} \mathrm{C}$ in the dark. Oocytes were then fixed as previously described, and immunocytochemistry was performed to assess anti-LAMP1 antibody labeling (Supplementary Table 1). Analysis was performed in the same manner as previously described, focusing on assessing lysosomal dilation using LAMP1. Lysosomal dilation indicated chloroquine treatment had effectively prevented lysosomal fusion with autophagosomes and inhibited autophagy (Mauthe et al., 2018). Oocyte viability was monitored visually post-treatment on live oocytes, and any other irregularities in morphology were captured and noted during imaging of fixed cells. Based on the results from initial dose-response experiments, a concentration of $200 \mu \mathrm{M}$ chloroquine was selected as the optimal treatment. This concentration was used to assess the impact of lysosomal inhibition on LC3B and EEA1 (Supplementary Table 1) immunolabeling within oocytes.

\section{Statistical Analyses}

All data were normalized to the young or control group and analyzed using a two-tailed unpaired Student's $t$-test or one-way ANOVA followed by a multiple comparisons test in GraphPad Prism 8.4.3. All experiments were performed with at least 3 biological replicates (specified for each set of experiments in results). Unless otherwise stated, all data are presented as mean \pm standard error of the mean (SEM). Statistical significance was considered as ${ }^{*} P<0.05$, or ${ }^{* *} P \leq 0.01$.

\section{RESULTS}

The initial goal of this study was to characterize the macroautophagy pathway in mouse pre-ovulatory oocytes. These oocytes were retrieved from young (4-6 week-old, equivalent to a human with initial fertility) and aged (12-14 month-old, equivalent to a human with declining fertility) mice. These age groups, from now on referred to as 'young' and 'aged,' were selected based on a pre-established model of oocyte aging within our laboratory. This model demonstrates decreased oocyte quantity and quality comparable to humans at their corresponding age time points (Camlin et al., 2017b).

\section{Relative Expression of Macroautophagy Marker Genes Map1/c3, Becn1, and Lamp1 Is Not Altered in Aged Mouse Oocytes}

To characterize the macroautophagy pathway in mouse pre-ovulatory oocytes, we initially focused on assessing the expression of the recognized autophagy markers Becn1, Map1lc3, and Lamp1. The expression of these markers was assessed in pre-ovulatory oocytes retrieved from young mice via RT-qPCR and normalized relative to the steady-state housekeeping gene, Ppia (Figure 3A). This strategy successfully amplified 


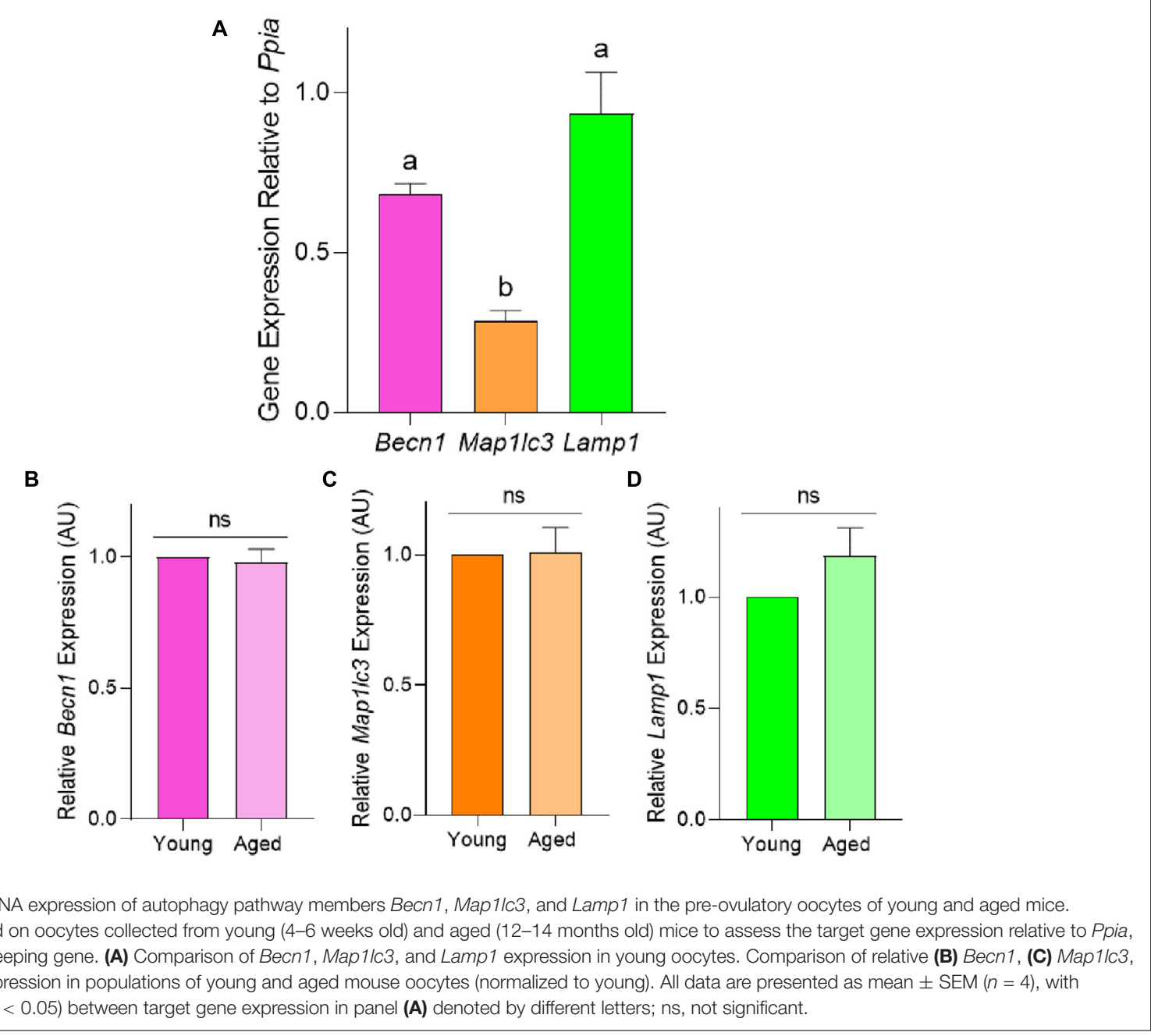

transcripts for each of the three gene targets, with Lamp1 proving to be the most highly expressed, followed by Becn 1 and Map1lc3. Notably, the relative expression of Map1lc3 proved significantly lower than Lamp1 $(p=0.0006)$ and Becn1 $(p=0.0148)$. In extending this analysis to focus on the expression of Becn1, Map1lc3, and Lamp1 genes in aged oocytes, there were no significant age-related differences in target transcript abundance (Figures 3B-D).

\section{Macroautophagy Related Proteins BECN1, LC3B-II, and LAMP1 Are Expressed at Equivalent Levels in Young and Aged Mouse Oocytes}

Immunoblotting confirmed the expression of BECN1, LC3B, and LAMP1 proteins in mouse oocytes. As shown in Figure 4, the three target proteins were readily detected in both young and aged populations of oocytes with minor non-specific cross-reactivity observed. Densitometric analysis of target band intensity revealed no significant differences in the abundance of BECN1, LC3B, or LAMP1 proteins in young and aged oocytes (Figure 4 and Supplementary Figure 3). Despite the detection of two bands denoting the presence of LC3B-I ( $19 \mathrm{kDa})$ and LC3B-II $(17 \mathrm{kDa})$ in whole ovarian protein, only the band corresponding to LC3B-II was identified in isolated oocytes (Figure 4B).

\section{BECN1 Immunolocalization Is Unchanged in Young and Aged Mouse Oocytes}

Having confirmed the presence of BECN1, LC3B, and LAMP1 in mouse oocytes, we next assessed the localization of these three proteins within oocytes using standard immunocytochemistry labeling techniques. Immunocytochemistry was accompanied by a specific image analysis method designed for this study to measure the number and area of all stained puncta captured within the cell. Standardization for this method was achieved by capturing $z$-stacked images through the middle $15 \mu \mathrm{m}$ of each oocyte (please refer to section "Materials and Methods"). This strategy allowed the assessment of subcellular localization and generated quantitative data of the number, size, and distribution of labeled vesicles within the sampled cell. It should be noted that quantitative data of puncta numbers does not necessarily reflect absolute values; however, this strategy allowed for comparative 

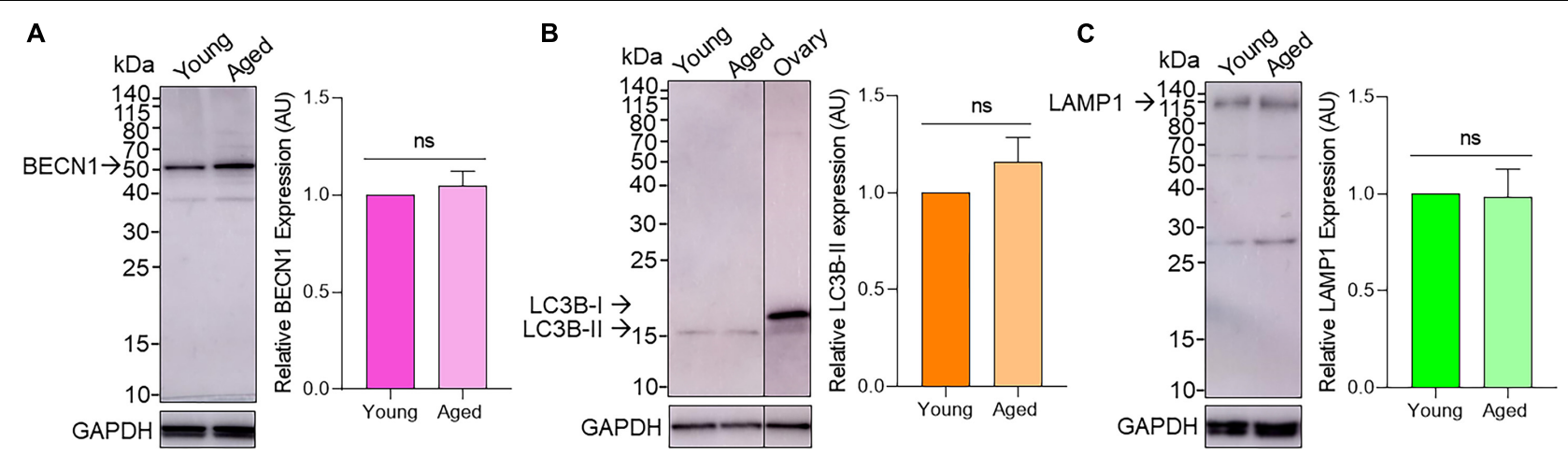

FIGURE 4 | Assessment of BECN1, LC3B, and LAMP1 protein expression in young and aged pre-ovulatory mouse oocytes. Oocyte protein lysates from young (4-6 weeks old) and aged (12-14 months old) mice were subjected to immunoblotting with (A) anti-BECN1 (52kDa), (B) anti-LC3B (19 and 17 kDa for LC3B-I and LC3B-II, respectively; including whole ovary positive control) or (C) anti-LAMP1 (120kDa) antibodies. Following detection of the target antigens, the blots were stripped and reprobed for the housekeeping protein GAPDH (35 kDa) to ensure equivalent protein loading in each lane. Densitometric analysis was performed on labeled bands corresponding to the predicted molecular weight of target proteins (please see arrows) to determine the protein abundance relative to GAPDH. Densitometric data are expressed as mean $\pm \operatorname{SEM}(n=4$, normalized to young) with statistical analysis revealing no significant (ns) differences in target protein expression between young and aged oocytes.

assessment of puncta numbers between groups. The details of which can yield important insight into the mechanics of the macroautophagy pathway.

Focusing first on BECN1, immunocytochemistry of young and aged oocytes revealed a distinct punctate distribution of this protein throughout the oocyte cytoplasm and nuclear domain (Figure 5A). Notably, the size distribution profile of the BECN1 labeled puncta proved to be highly variable within both young and aged oocytes. In this context, $98 \%$ of puncta ranged between 0 and $2 \mu \mathrm{m}^{2}$. In assessing puncta $>2 \mu \mathrm{m}^{2}$, we noted a reciprocal relationship whereby the larger the puncta area, the fewer the number of puncta detected in that size range (Figure 5B). Despite this variability, we did not record any significant changes in puncta size profiles between the young and aged oocyte groups. Notable trends in our data included an increased number of large puncta greater than $10 \mu \mathrm{m}^{2}$ in aged oocytes, accompanied by the accumulation of these puncta toward the periphery of the aged cells (Figures 5C,D). The latter was assessed by calculating the percentage of puncta residing in the outer $10 \mu \mathrm{m}$ diameter of the oocyte cytoplasm.

\section{Accumulation of Large LC3B Labeled Puncta in Aged Mouse Oocytes}

Immunocytochemical labeling of oocytes with anti-LC3B antibodies revealed a similar localization profile to BECN1, with punctate staining distributed throughout the cell cytoplasm (Figure 6A). Similarly, the size distribution of LC3B labeled puncta demonstrated a comparable trend to that of BECN1, with a large proportion of the puncta identified between 0 and $2 \mu \mathrm{m}^{2}$. Once again, the proportion of LC3B labeled puncta decreased as their area increased. Unlike BECN1, however, significant differences were observed in the distribution of LC3B puncta across several size categories when comparing young and aged oocytes (Figure 6B). The specific size categories of interest included those less than $0.196 \mu \mathrm{m}^{2}$, distributed between 0.196 and $1.767 \mu \mathrm{m}^{2}$, and those greater than $10 \mu \mathrm{m}^{2}$ (Figure 6B; shaded areas). These categories pertain to the predicted sizes of specific biological structures that contain LC3B. Puncta within the category of less than $0.196 \mu^{2}$ are likely to correspond to phagophores, immature double-membrane structures that grow to form autophagosomes. This particular size group experienced a significant age-related reduction $(p=0.001)$ in the older oocyte population (Figure 6C). The next size category, 0.196-1.767 $\mu \mathrm{m}^{2}$, corresponds to the documented size of autophagosomes [0.5-1.5 $\mu \mathrm{m}$ in diameter (Mizushima et al., 2002)], and also displayed a significantly decreased number of puncta $(p=0.011)$ in aged oocytes (Figure 6D). Conversely, aged oocytes were characterized by a significant accumulation $(p=0.009)$ of puncta greater than $10 \mu \mathrm{m}^{2}$. Moreover, these larger puncta were significantly more likely to reside within the periphery (outermost $10 \mu \mathrm{m}$ ) of the aged oocyte cytoplasm $(p=0.005)$ (Figures 6E,F). This differential localization and large size compared to that of a typical autophagosome prompted a more detailed characterization of the identity of this latter class of LC3B labeled puncta (as described below).

\section{Large LC3B Positive Puncta Identified as Amphisomes Containing EEA1 in Mouse Oocytes}

We next sought to determine the identity of LC3B-staining puncta greater than $10 \mu \mathrm{m}^{2}$ and further characterize the changes observed in these puncta between aged and young oocyte groups. Alternative structures containing LC3B include intracellular vesicles that fuse with autophagosomes, such as lysosomes (to form autolysosomes) and endosomes (to form amphisomes) (Klionsky et al., 2014). To explore the possibility of these large puncta being amphisomes, immunocytochemistry experiments were employed to co-localize LC3B with the recognized endosome marker, early endosome antigen 1 (EEA1). The LC3B and EEA1 markers were revealed to highly co-localize within the 


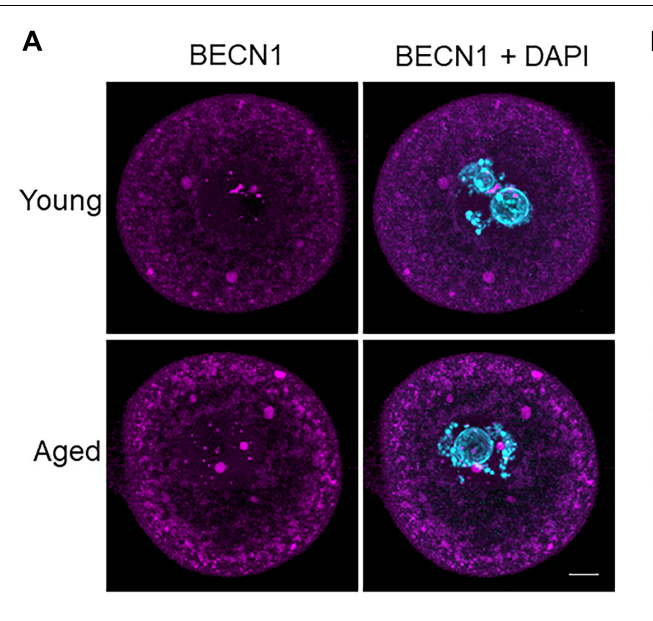

C

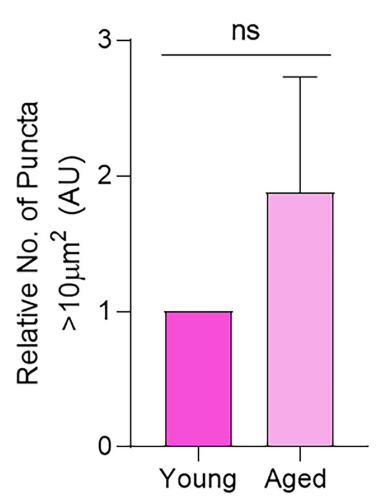

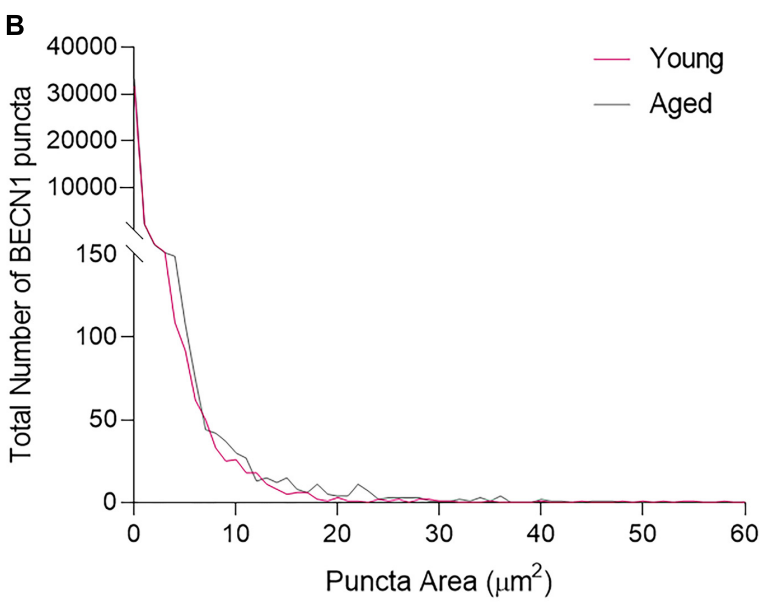

D

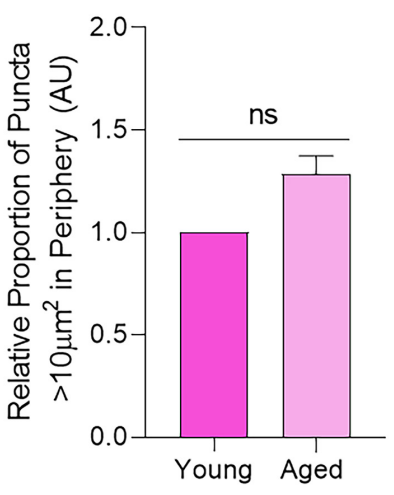

FIGURE 5 | BECN1 protein localization and puncta distribution in young and aged mouse oocytes. Oocytes from young (4-6 weeks old) and aged (12-14 months old) mice were fixed prior to labeling with anti-BECN1 antibodies (pink), nuclear counterstaining with DAPI (cyan), confocal imaging (60x objective), and puncta analysis. (A) Representative images are presented to illustrate the punctate cytoplasmic and nuclear localization of BECN1 in young and aged oocytes. Scale bar $=10 \mu \mathrm{m}$. (B) Images were assessed for the number and area of BECN1 labeled puncta, and these data were used to graph the size distribution of puncta in populations of young (pink line) and aged oocytes (gray line). (C) Comparison of the number of BECN1 labeled puncta $>10 \mu \mathrm{m}^{2}$ in young and aged oocytes (normalized to young). (D) Comparison of the proportion of BECN1 labeled puncta $>10 \mu \mathrm{m}^{2}$ that resided within the periphery (outermost $10 \mu \mathrm{m}$ ) of the oocyte (normalized to young). $N=3$, ns, not significant.

large puncta (Figure 7A). The degree of this co-localization was determined using Pearson's correlation coefficient illustrating equivalent distribution patterns in both young $(R=0.7686)$ and aged groups $(R=0.7618)$. To further confirm the presence of these two proteins within the same structures, a proximity ligation assay (PLA) was used. Notably, PLA produces a fluorescent signal wherever the epitopes of the target proteins reside within a maximum of $40 \mathrm{~nm}$. In both young and aged oocytes, a strong PLA signal was displayed in a similar pattern to the co-localization experiments (Figure 7B). Combined, these data confirm that the large immunostained puncta (i.e., greater than $10 \mu \mathrm{m}^{2}$ ) were likely to be amphisomes.

\section{Lysosome Number Decreases in Aged Mouse Oocytes}

Subsequently, the third macroautophagy pathway marker, lysosomal-associated membrane protein 1 (LAMP1), was also assessed through immunocytochemistry. The staining of LAMP1 displayed a dispersed, punctate distribution, reflective of lysosomal staining within a cell (Figure 8A). As with BECN1 and LC3B, the total distribution of the puncta number and area was first assessed. While lysosome size varies under physiological conditions, literature pertaining to somatic cells suggests lysosomes usually occupy a size range of between 0.03 and $0.5 \mu \mathrm{m}^{2}$ (Ponsford et al., 2020; Trivedi et al., 2020). In the present study, a large number of LAMP1 puncta were observed within this range (Figure $\mathbf{7 B}$ shaded area), and the number of puncta decreased as area increased (Figure 8B). Notably, lysosomes can increase in size due to fusion with endosomes, phagosomes, or autophagosomes, giving rise to structures that exceed the $0.03-0.5 \mu \mathrm{m}^{2}$ range (Luzio et al., 2007). This shift in size may be reflected in our data set with fewer LAMP1 labeled puncta detected up to $4 \mu \mathrm{m}^{2}$ in area (Figure 8B). Overall, there were clear differences when comparing the size distribution of lysosomes in young and aged oocytes, contributing to a $30 \%$ decrease in lysosomes in aged oocytes $(p=0.01)$ (Figure 8C). Observing specific areas of interest within the size distribution, 


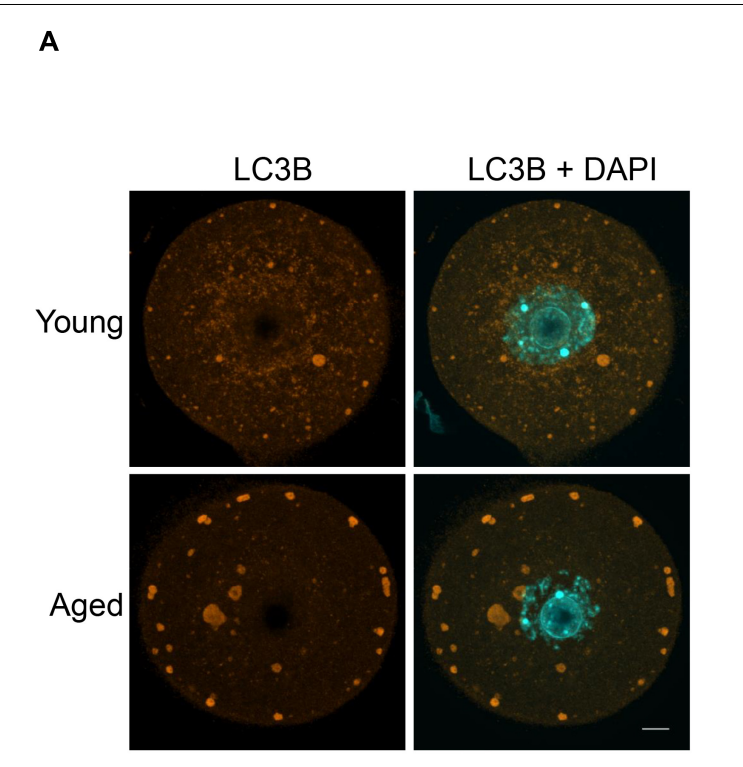

C

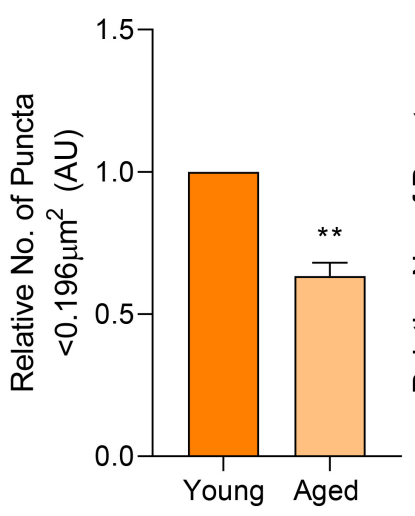

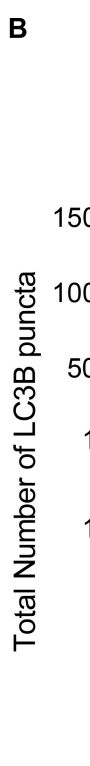

D

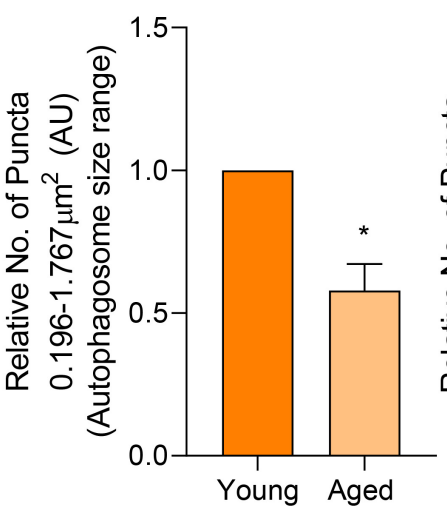

E

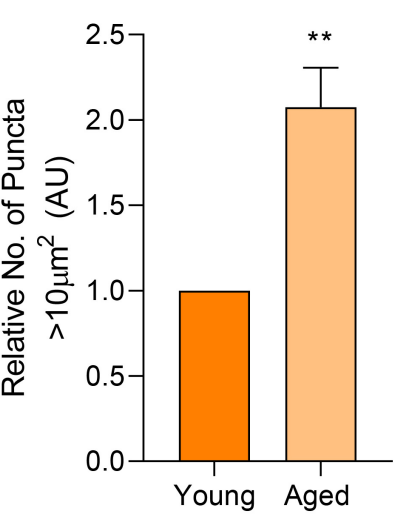

$\mathbf{F}$

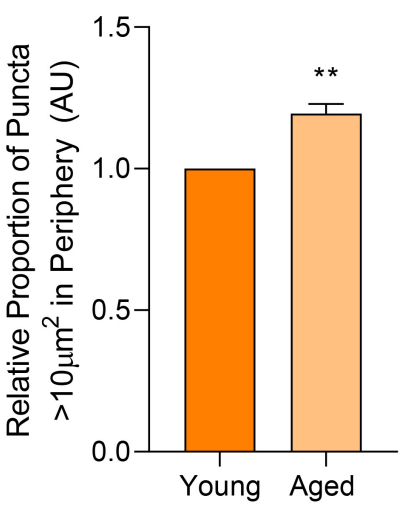

FIGURE 6 | LC3B protein localization and puncta distribution in young and aged mouse oocytes. Oocytes from young (4-6 weeks old) and aged (12-14 months old) mice were fixed prior to labeling with anti-LC3B antibodies (orange), nuclear counterstaining with DAPI (cyan), confocal imaging (60x objective), and puncta analysis. (A) Representative images illustrate the punctate cytoplasmic localization of LC3B in young and aged oocytes. Scale bar = $10 \mu \mathrm{m}$. (B) Images were assessed for the number and size of LC3B labeled puncta, and these data were used to graph the size distribution of puncta in populations of young (orange line) and aged oocytes (gray line). The puncta size groupings that differed significantly in abundance between young and aged oocytes are highlighted in red, orange, and yellow shading. (C) Comparison of the number of LC3B labeled puncta $<0.196 \mu \mathrm{m}^{2}$, (D) 0.196-1.767 $\mu \mathrm{m}^{2}$ [documented area of autophagosome (Mizushima et al., 2002)], (E) and $>10 \mu \mathrm{m}^{2}$ in young and aged oocytes (normalized to young). (F) Comparison of the proportion of LC3B labeled puncta $>10 \mu \mathrm{m}^{2}$ that resided in the periphery (outermost $10 \mu \mathrm{m}$ ) of the oocyte (normalized to young). $N=3,{ }^{\star} P<0.05,{ }^{\star \star} P \leq 0.01$.

the total decrease in lysosomes was primarily attributed to a decrease in lysosomes ranging from 0.03 to $0.5 \mu \mathrm{m}^{2}$ in aged oocytes $(p=0.01)$ (Figure 8D).

\section{Lysosomal Inhibition Promotes the Formation of Large Amphisomes in Mouse Oocytes}

To explore the mechanism(s) by which an accumulation of amphisomes may occur in aged oocytes, an in vitro model was established in which oocytes from young mice were incubated with chloroquine. Chloroquine is an inhibitor of lysosomal activity that effectively prevents the fusion of lysosomes with autophagosomes and increases lysosomal area (Mauthe et al., 2018). Initially, effective chloroquine concentrations were titrated to encompass doses between 25 and $400 \mu \mathrm{M}$ that were previously used in somatic cell and oocyte studies (Pellegrini et al., 2014; Jia et al., 2018; Mauthe et al., 2018; Mihalas et al., 2018; Miao et al., 2019). Specifically, oocytes were co-incubated with chloroquine $(25-400 \mu \mathrm{M})$ for $6 \mathrm{~h}$, after which LAMP1 immunolocalization was assessed to measure lysosomal area and number. Importantly, all treatment groups displayed comparable viability $(>89 \%)$ and cellular morphology to the population of untreated control oocytes except for those exposed to the 

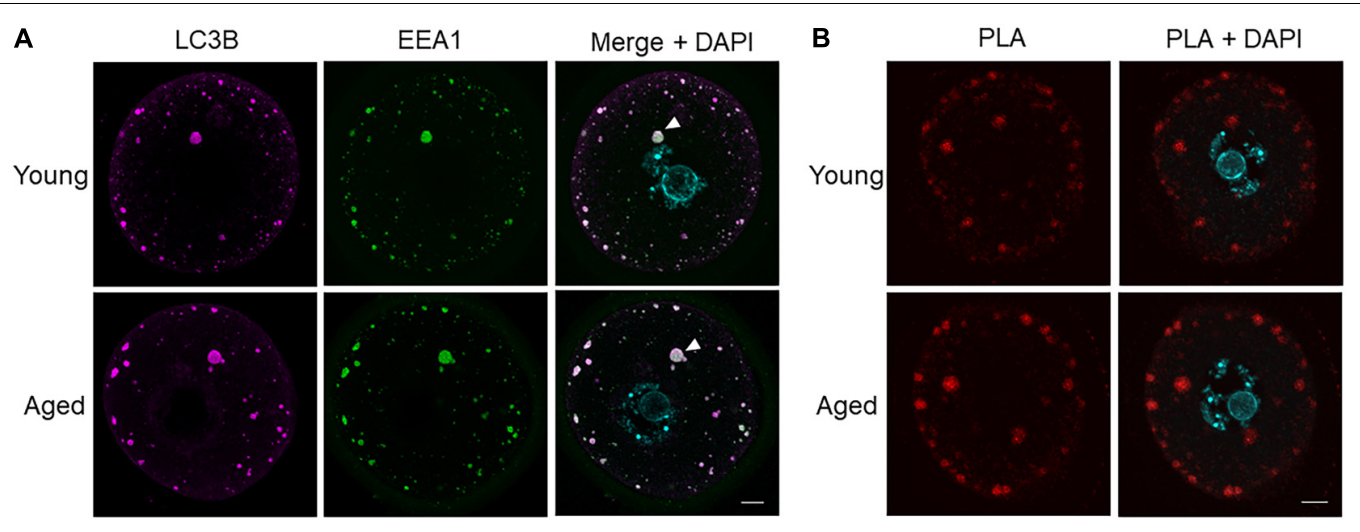

FIGURE 7 | Co-localization and proximity ligation assay for LC3B and EEA1 demonstrating presence of amphisomes in young and aged mouse oocytes.

(A) Oocytes from young (4-6 weeks old) and aged (12-14 months old) mice were fixed prior to labeling with anti-LC3B antibodies (pink), anti-EEA1 antibodies (green), nuclear counterstaining with DAPI (cyan), confocal imaging (60x objective), and puncta analysis. Representative images illustrate the punctate cytoplasmic co-localization of LC3B and EEA1 in young and aged oocytes where white arrow points to an example of large puncta. Scale bar = $10 \mu \mathrm{m}, \mathrm{N}=4$. (B) Fixed young and aged oocytes underwent a proximity ligation assay for LC3B and EEA1 and were imaged through confocal microscopy (60× objective). Representative images illustrate the characteristic punctate staining similar to that in (A) where red signal indicates proteins are within $40 \mathrm{~nm}$.

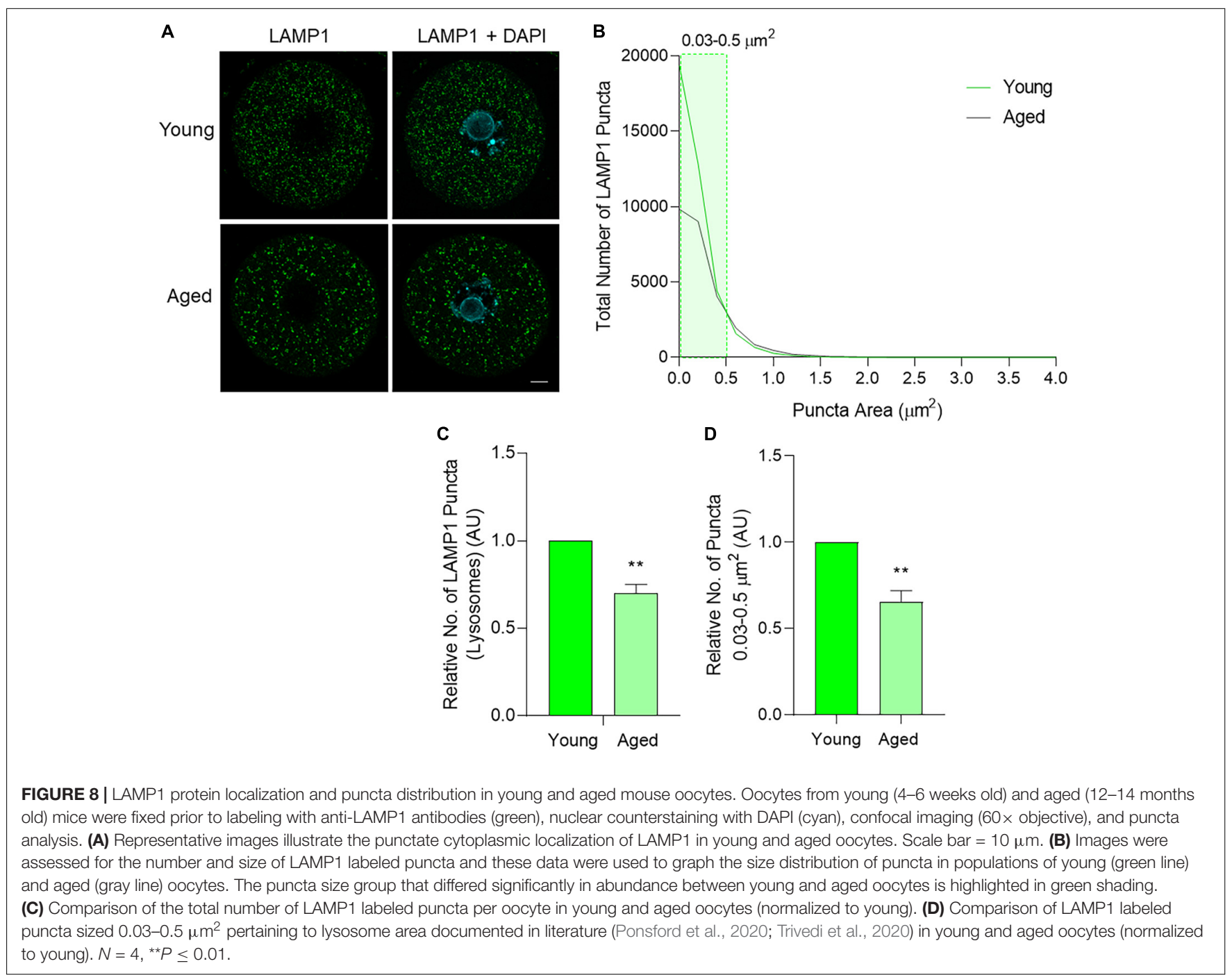



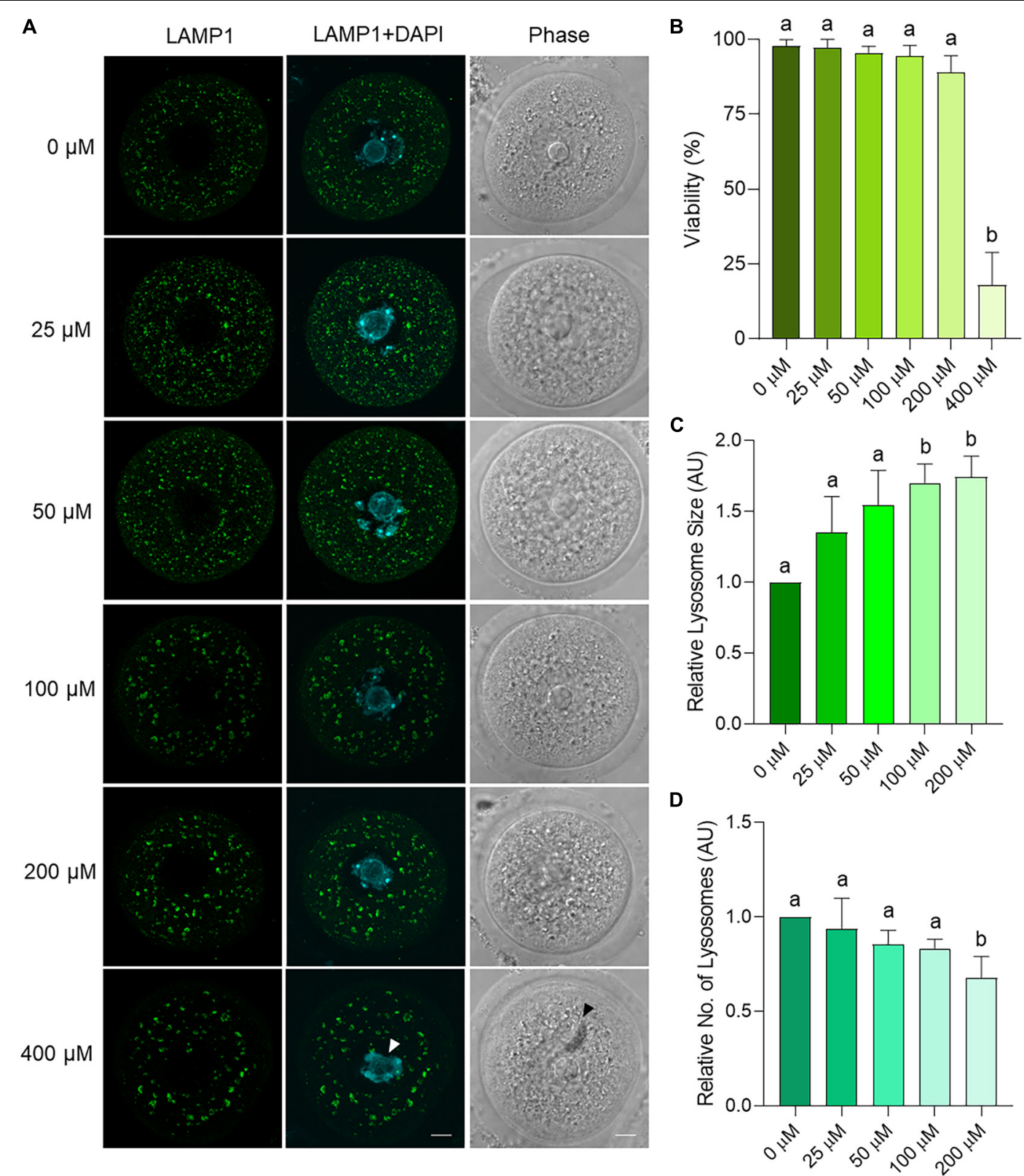

FIGURE 9 | Dose response trial using chloroquine on young mouse oocytes to assess effects on lysosome parameters. Oocytes from young (4-6 weeks old) mice were treated for $6 \mathrm{~h}$ with varying concentrations of chloroquine before fixation and labeling with anti-LAMP1 antibodies (green), nuclear counterstaining with DAPI (cyan), confocal imaging (60x objective), and puncta analysis. Scale bar $=10 \mu \mathrm{m}$. (A) Representative images illustrate the changes in size and number of LAMP1 labeled puncta within oocytes treated with $0,25,50,100,200$, and $400 \mu \mathrm{M}$ chloroquine. White arrow indicates reduced chromatin condensation and black arrow indicates cytoplasmic inclusion. (B) Viability of oocytes as the percentage of live, morphologically normal oocytes in each treatment group. (C) Average area and (D) number of lysosomes (LAMP1-staining puncta) as determined through puncta analysis. Data is normalized to the untreated control. Statistical significance $(P<0.05)$ between groups is indicated by different letters, $n=3$ for all groups except 0 and $100 \mu \mathrm{M}$ with $n=6$. Statistics not performed on $400 \mu \mathrm{M}$ due to low viability.

highest chloroquine dose of $400 \mu \mathrm{M}(p<0.0001)$ (Figure 9B). This dose resulted in abnormal oocyte morphology, reduced DNA condensation, and the formation of large cytoplasmic inclusions indicating significant toxicity and was excluded from further analysis (Figure 9A arrows). All other concentrations of chloroquine yielded a dose-dependent increase in lysosome area, with this response achieving statistical significance at concentrations of 100 and $200 \mu \mathrm{M}$ chloroquine $(p=0.0048$ and 0.0150, respectively) (Figures 9A,C). Lysosomal number also significantly decreased at $200 \mu \mathrm{M}(p=0.0257)$ (Figure 9D). Due to the $200 \mu \mathrm{M}$ chloroquine treatment having the highest efficacy, this concentration was selected for use in further experiments.

After optimizing this lysosomal inhibition strategy, oocytes treated with $200 \mu \mathrm{M}$ chloroquine for $6 \mathrm{~h}$ were assessed 


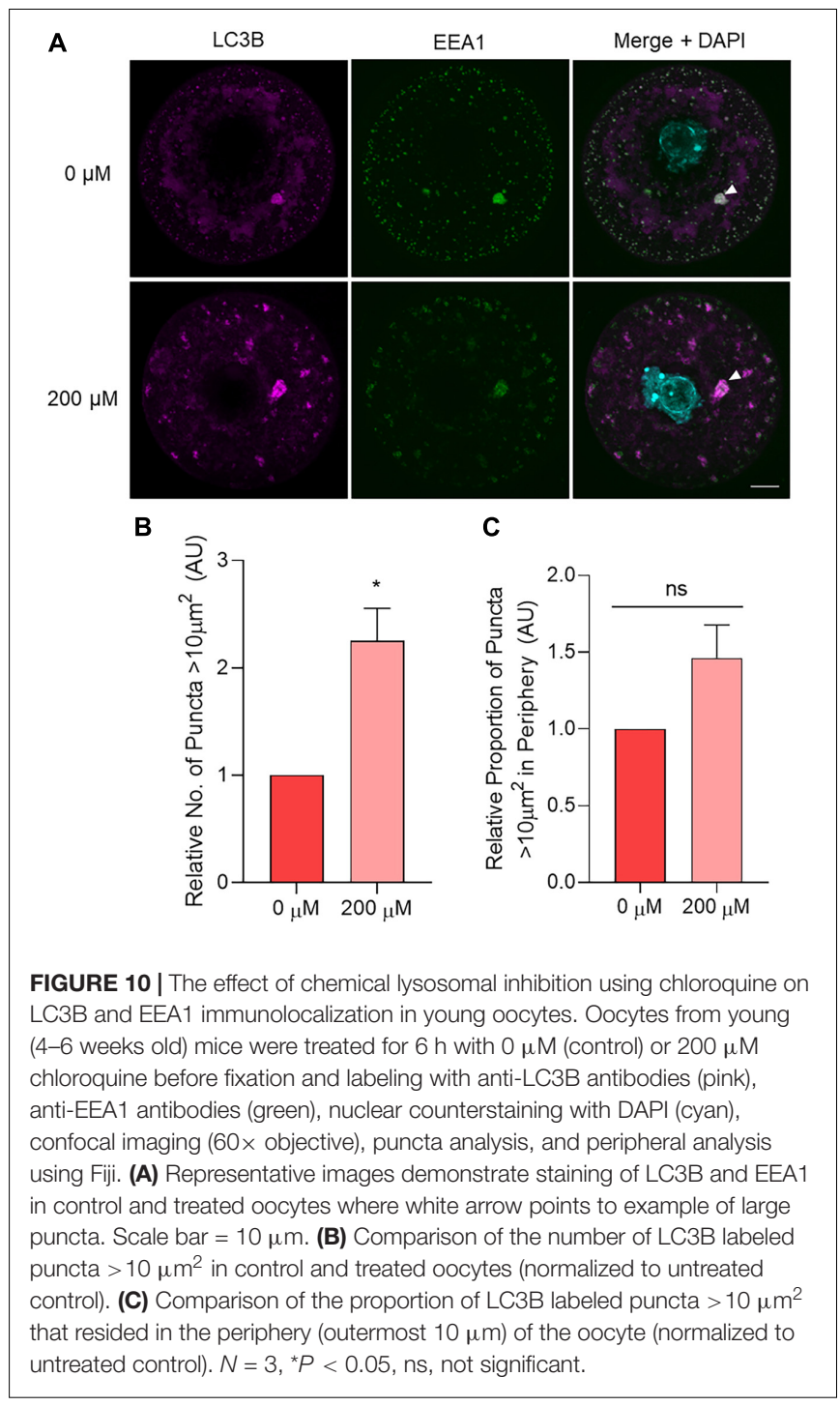

for immunolabeling patterns of LC3B, EEA1, and LAMP1. LAMP1 was used to ensure the consistency of inhibitor-induced lysosomal dilation across replicates (Supplementary Figure 4). Assessing $\mathrm{LC} 3 \mathrm{~B}$ and EEA1 staining, a punctate distribution featuring irregularly shaped, less rounded puncta (potential amphisomes) was observed in chloroquine treated oocytes (Figure 10A). Similarly to aged oocytes, a significant increase in the number large puncta greater than $10 \mu \mathrm{m}^{2}$ was evident in the $200 \mu \mathrm{M}$ chloroquine treatment group (Figure 10B). Following this, the colocalization of LC3B and EEA1 within these large puncta (i.e., area $>10 \mu \mathrm{m}^{2}$ ) was quantified. This colocalization analysis revealed a highly significant $(p=0.0016), 38 \%$ reduction in the dual-labeling of these markers in chloroquine challenged oocytes compared to the untreated control $(R=0.515$ and 0.829 , respectively). While the localization of the large puncta tended to increase in the periphery of the treated cells compared to controls cells, this did not yield a significant difference $(p=0.0987)$ (Figure 10C). Combined, these results provide a mechanistic link between the compromise of lysosomal function and the accumulation of amphisomes characteristic of naturally aged oocytes (Figure 11).

\section{DISCUSSION}

Aging oocytes experience a decrease in quality, eventuating in poor developmental outcomes for the subsequent embryo (te Velde and Pearson, 2002; Doherty and Pal, 2011). Despite continuing efforts to pinpoint critical mechanisms involved in the loss of oocyte quality, there has been minimal progression in fertility treatment and preservation for women of advanced reproductive age. Macroautophagy is an essential protein degradation pathway that decreases in efficiency in aging somatic cells (Terman, 1995; Vilchez et al., 2014; Peters et al., 2020). However, few studies have sought to investigate the contribution of this pathway to the age-dependent deterioration of oocyte quality. In recognizing this knowledge gap, we have used comprehensive image analysis techniques to demonstrate that the localization of crucial macroautophagy pathway markers is modulated with age in the pre-ovulatory mouse oocyte. Moreover, we established a novel short-term treatment model that recapitulates the hallmark features of macroautophagy found in aged oocytes, revealing decreased lysosome abundance as the mechanism underpinning these changes.

Upon comparison of the three autophagy pathway members Becn1/BECN1, Map1lc3/LC3B, and Lamp1/LAMP1, no gross changes in gene or protein expression were detected between young and aged oocyte groups. This indicated that the overall expression of autophagy pathway members remains consistent in pre-ovulatory oocytes throughout the aging process. LC3B proteins exist in two forms within the cytoplasm; LC3B-I, which exists as free protein in the cytoplasm, and LC3B-II, the lipidated protein within the autophagosome membrane (Klionsky et al., 2016). In our study, only LC3B-II was detected in oocytes indicating that the LC3B within the oocyte was bound within autophagosomes. By contrast, both forms of LC3B were detected in whole ovarian tissue lysates. One possible explanation for this is that LC3B-I could be expressed in the somatic cells surrounding the oocyte, whereby LC3B-I is converted to LC3BII and then transported to the oocyte. In line with this, it is known that oocytes possess transzonal projections enabling the surrounding granulosa cells to transport molecules to the oocyte cytoplasm to aid function and growth (Gilchrist et al., 2008). This generally low abundance of LC3B may also be linked to its relative gene expression, where we found Map1lc3 exhibited significantly lower expression than that of Becn1 and Lamp1. Additionally, the low abundance and expression of Map1lc3/LC3B could be indicative of an autoregulatory 'brake' mechanism. This autoregulatory 'brake' can occur when upstream members of the pathway, including mammalian target of rapamycin (mTOR) and death-associated protein 1 (DAP1), are activated to prevent overactivation of autophagy (Koren et al., 2010). This mechanism has been demonstrated to occur under conditions of nutrient deprivation and rapamycin treatment (pharmacological activation) (Koren et al., 2010); however, it is yet to be determined if this mechanism occurs in the oocyte. 


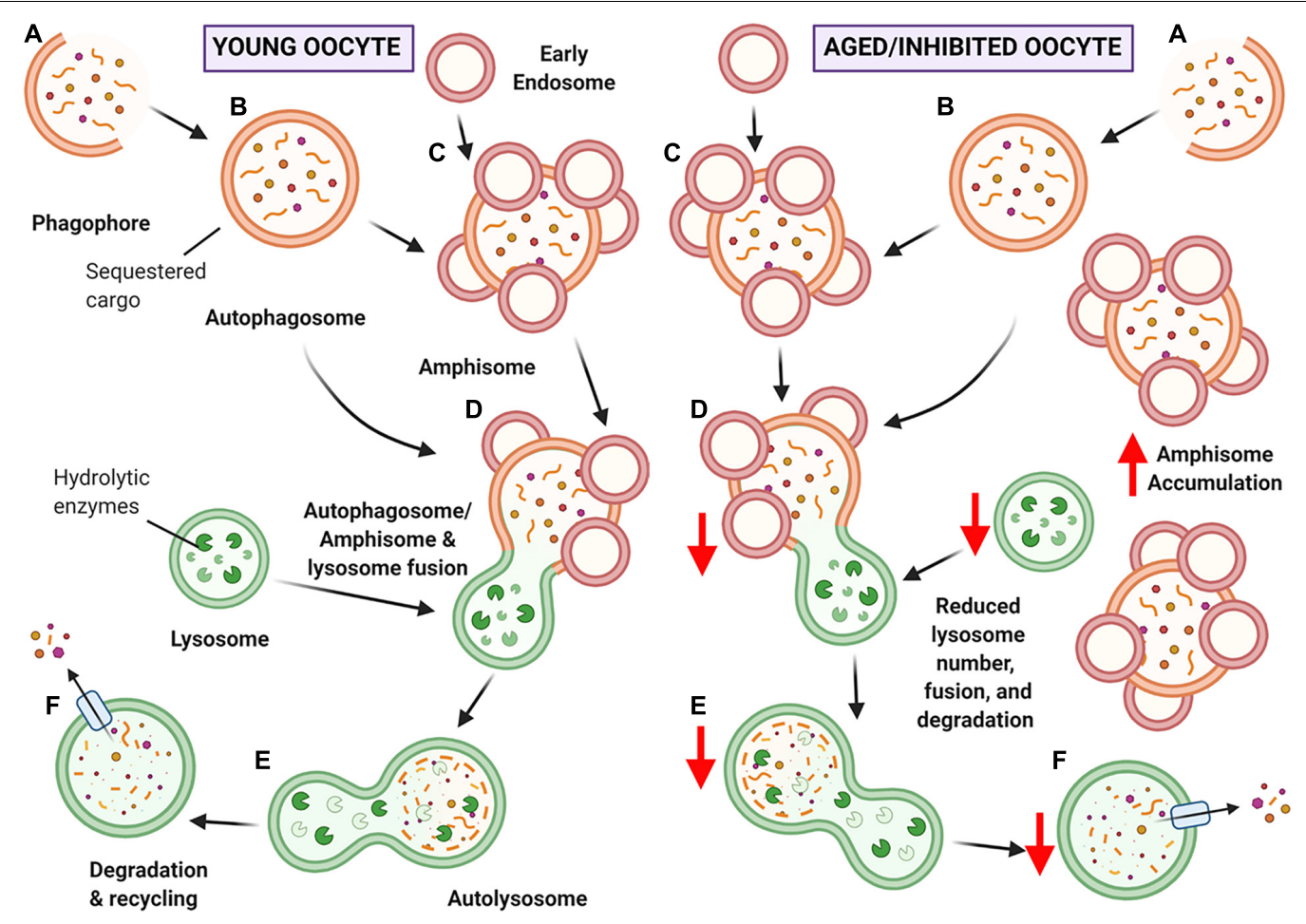

FIGURE 11 | Schematic of hypothesized 'bottleneck model' of macroautophagy in aging or chloroquine treated oocytes. (A) Macroautophagy begins with the formation of the phagophore, and as it continues to grow and sequester cargo, it forms a completed double-membraned vesicle termed the autophagosome, encompassing the cargo to be degraded (B). Based on our data, the autophagosome may then fuse with early endosomes to create amphisomes (C). The autophagosome or amphisome can then fuse with the acidic lysosome containing hydrolytic enzymes (D) to form an autolysosome (E) in which the encapsulated cargo is broken down into its basic building blocks. Lastly, the broken down cargo is extruded back into the cell cytoplasm for recycling (F) (Feng et al., 2014; Klionsky et al., 2016). In young oocytes (left-hand panel) this process is hypothesized to occur normally. In aged or chloroquine treated oocytes (right-hand panel), the availability of lysosomes is decreased, resulting in less lysosomal fusion with autophagic vesicles and ultimately reduced protein degradation. Due to the bottleneck created by the reduced lysosomal availability (due to decreased numbers in aging or loss of functionality with inhibition) an accumulation of large amphisomes is observed. Created with BioRender.com.

BECN1 is a component of the phosphoinositide 3-kinase (PI3K) nucleation complex involved in activating macroautophagy and is a marker commonly used to monitor autophagy (Klionsky et al., 2016). However, there is increasing evidence that BECN1 is also involved in non-autophagy-related pathways (Martinet et al., 2013; Myung Park et al., 2014; Xu et al., 2017). Immunocytochemistry experiments within this study demonstrated high variability of BECN1 puncta localization in the cytoplasm and nucleus within all oocytes regardless of their age group. This nuclear localization may be explained by the involvement of Becnl in DNA double-stranded break repair pathways. This involvement has been demonstrated in studies assessing radiation-induced DNA damage where Becn1 has been implicated in regulating DNA double-strand break repair mechanisms (Myung Park et al., 2014; Xu et al., 2017). Due to this association of BECN1 with non-autophagyrelated DNA double-strand break repair pathways that become dysfunctional with aging in the oocyte (Titus et al., 2013; Turan and Oktay, 2020), this commonly used marker was not used in subsequent experiments.

In contrast, LC3B displayed clear punctate cytoplasmic localization with a differing expression between young and aged oocytes. Subsequently, LC3B, in combination with LAMP1, was employed as a primary marker of macroautophagy in the remainder of this study. LC3B is a reliable and widely used maker of autophagy due to its role as a component of the autophagosome membrane (Klionsky et al., 2016). It has previously been suggested that a decline in autophagy efficiency due to declined autophagosome numbers in aging somatic cells arises from decreased formation and elimination of autophagosomes (Cuervo et al., 2005; Wilhelm and Richly, 2018). This decrease in the formation of autophagosomes is reflected in our dataset, whereby a reduction in the number of autophagosomes (0.196-1.767 $\mu \mathrm{m}^{2}$ category) and phagophores $\left(<0.196 \mu \mathrm{m}^{2}\right.$ category) was observed in aged oocytes compared to their young counterparts. Accompanying this, an increase in large puncta greater than $10 \mu \mathrm{m}^{2}$ was evident. These large puncta were subsequently identified as amphisomes through the co-localization of LC3B and EEA1, an early endosome marker. The accumulation of these large amphisomes, accompanied by a concomitant decrease in autophagosomes, was a key finding within this study. Amphisome accumulation has also been observed in other cell types, including aging neurons in Alzheimer's disease and hepatocytes exposed to leupeptin, a 
protease inhibitor that prevents lysosomal protein degradation (Berg et al., 1998; Tammineni and Cai, 2017).

The accumulated amphisomes documented in our study were substantially larger than typical autophagosomes $\left(0.196-1.767 \mu \mathrm{m}^{2}\right)$ and endosomes $\left(0.071-0.785 \mu \mathrm{m}^{2}\right)$ (Mizushima et al., 2002; Skjeldal et al., 2012). Amphisomes can comprise early and late endosomes fused with autophagosomes and can undergo multiple independent fusions with other endosomes (Berg et al., 1998). Additionally, defects in the endocytic pathway can cause early endosome enlargement (Kaur and Lakkaraju, 2018). By way of example, Ras-associated binding protein RAB5, a GTPase that controls the fusogenic properties of early endosomes, can be altered to a mutant form known as RAB5(Q79L). This mutant RAB5(Q79L) causes endosomes to retain early endosome markers as they mature and become 'giant endosomes' (Wegner et al., 2010). These structures retain the ability to fuse with other such endosomes, leading to a dramatic expansion in vesicle size. In a similar study, the overexpression of Rab5 led to an increased endosome size of up to $10 \mu \mathrm{m}$ in diameter (Skjeldal et al., 2012). In this way, these mechanisms provide viable explanations for how oocyte amphisomes may reach the large sizes documented here. Overall, our observations are the first of amphisomes in aging oocytes that are larger than those observed naturally occurring in other cells, indicating that oocyte aging presents a challenge to the normal autophagic response.

It is also noteworthy that large amphisomes were typically localized to the periphery of aged oocytes. In seeking an explanation for this observation, it is known that the movement of intracellular vesicles, including amphisomes, autophagosomes, and lysosomes is reliant on the cytoskeleton network (Kamal and Goldstein, 2000; Nakamura and Yoshimori, 2017). The directional movement of vesicles is determined by various microtubule motor proteins that move toward the periphery (e.g., kinesin superfamily proteins KIF5 and KIF3) or perinuclear region (e.g., KIFC1, 2, and 3) depending on the plus or minus orientation of the microtubules to which they attach (Hirokawa et al., 2009; Camlin et al., 2017a; Muralidharan and Baas, 2019). A recent study assessing the microtubule motor control of early endosomes in human endothelial cells demonstrated that simultaneous silencing of KIFC1 and stromal interaction molecule 1 (STIM1) resulted in more peripherally distributed EEA1 positive early endosomes (Villari et al., 2020). Aligning with this, Kifc1 gene and KIFC1 protein expression decreases in the aged oocyte, with functional siRNA-mediated knockdown and pharmacological inhibition of Kifc1/KIFC1 in young oocytes recapitulating hallmarks of poor oocyte quality such as increased aneuploidy rates (Mihalas et al., 2019). Taken together, these findings provide a putative explanation for the peripheral distribution of amphisomes noted in our study and provide the impetus to investigate the role of KIFC1 and other KIF protein members in facilitating macroautophagy in the aging oocyte.

Here, it was hypothesized that the observed increase in amphisomes in aged oocytes was attributed to decreased rates of lysosomal clearance. Consistent with this notion, the assessment of LAMP1 puncta as a proxy for lysosomes revealed a pronounced decrease in lysosome number among aged oocytes. It should be noted that this decrease was restricted to puncta within the size range pertaining to lysosomes $(0.03-$ $\left.0.5 \mathrm{\mu m}^{2}\right)$. By contrast, larger LAMP1 labeled structures of up to $4 \mu \mathrm{m}^{2}$ were present in both young and aged oocytes. It is possible that the persistence of these larger LAMP1containing structures may account for the detection of equivalent amounts of LAMP1 protein (as assessed via densitometric analysis of LAMP1 immunoblots) across young and aged oocyte groups. Although the cause of the age-related decrease in lysosomes is yet to be established, we postulate at least two possible mechanisms by which this phenomenon may occur. Firstly, oxidative insults arising under conditions of cellular oxidative stress may compromise the integrity of the lysosomal membranes and promote lysosomal rupture (Brunk et al., 1997). In agreement with this model, chronic oxidative stress is a well-established driver of oocyte aging (Goud et al., 2008). If this were to hold true, the rupture of lysosomes could exacerbate oxidative damage within oocytes and contribute to the presentation of additional, functionally harmful hallmarks of aging such as lipofuscin accumulation (Brunk et al., 1997). An alternative explanation for decreased lysosomal number lies upstream in the biogenesis of these organelles. In this context, several lysosome biogenesis-related pathways share transcriptional control under the transcription factor EB (TFEB) (Saftig and Klumperman, 2009; Bajaj et al., 2019). It follows that TFEB dysregulation contributes to the pathogenesis of lysosomal storage diseases and neurodegenerative diseases associated with aging (Sardiello, 2016; Bajaj et al., 2019; Cortes and La Spada, 2019). This age-associated dysregulation of TFEB in long-lived neuronal cells can disrupt lysosome biogenesis and reduce lysosome number (Cortes and La Spada, 2019); while yet to be elucidated, this mechanism could be a potential contributor to reduced lysosome number in oocytes. Irrespective of the mechanism, the decreased prevalence of lysosomes within aged oocytes could conceivably create a bottleneck effect leading to an upstream accumulation of amphisomes within these cells. Ultimately, these observations may reflect a reduction in the efficiency of protein degradation and hence a delay in the removal of damaged or dysfunctional protein cargo contained within the amphisomes. This could compromise oocyte maturation with potential repercussions for post-fertilization zygotic development, a possibility that warrants further investigation.

In order to model decreased lysosome availability, the lysosomal inhibitor chloroquine was used to prevent the fusion of autophagosomes and lysosomes (Mauthe et al., 2018), mimicking the bottleneck effect described above. Notably, oocytes treated with $200 \mu \mathrm{M}$ chloroquine displayed an increased number of amphisomes greater than $10 \mu \mathrm{m}^{2}$, revealing that amphisome accumulation in the aged oocyte is likely linked to reduced lysosome availability. In the presence of chloroquine, amphisome morphology was characterized by irregularly shaped structures and a reduced abundance of embedded EEA1. These features varied substantially from the uniform amphisomes present in untreated control oocytes. In addition to lysosomal accumulation, chloroquine can also accrue 
within acidic endosomal organelles, leading to perturbation of their acidity, increased osmotic pressure, and eventual membrane rupture; responses that have been exploited in the context of drug delivery and release within target cells (Yu et al., 2016; Hajimolaali et al., 2021). Given this insight, it is possible that chloroquine action within the oocyte may be attributed to a compromise of endosome integrity, accounting for the irregular amphisome morphology witnessed after chloroquine challenge. While chloroquine treatment successfully modeled the physiological mechanism(s) responsible for amphisome accumulation in aged oocytes, it is unlikely to mirror all aspects of macroautophagy compromise due to the multifaceted, complex nature of aging. Nevertheless, this treatment regimen did replicate a key feature of macroautophagy in the aging oocyte by preventing damaged and dysfunctional proteins from being cleared from the cells. In this way, amphisome accumulation caused by decreased lysosomal efficiency could be a symptom of dysfunctional autophagy contributing to the age-dependent deterioration of oocyte quality. This treatment regimen may prove valuable for future investigation into the mechanisms underlying altered autophagy pathway responses during female reproductive aging. Specifically, it could shed light on the behavior of upstream members of the macroautophagy pathway and aid in assessing the effects of reduced lysosomal efficiency and amphisome accumulation during oocyte development and maturation. Ultimately these investigations will contribute to the development of novel prophylactic drugs that could be used to prevent lysosomal decline throughout oocyte aging and hence maintain oocyte quality.

In summary, this study is the first to utilize imagebased analysis to discern signature changes occurring in the macroautophagy pathway in maternally aged oocytes, revealing fundamental changes that could contribute to reduced oocyte quality. Among the key changes documented were age-dependent reductions in the number of autophagosomes and lysosomes and a reciprocal accumulation of amphisomes, all of which have significant functional implications for oocyte proteostasis. Furthermore, we have demonstrated that amphisome accumulation can be induced via the inhibition of lysosome activity in oocytes, providing a robust model of macroautophagy degeneration in aging oocytes. Collectively, these data demonstrate a role for macroautophagy in the maintenance of pre-ovulatory oocyte health and provide the impetus to focus on therapeutic interventions that modulate this pathway to promote oocyte longevity and combat the age-related decline in female fertility.

\section{REFERENCES}

Agarwal, A., Gupta, S., and Sharma, R. K. (2005). Role of oxidative stress in female reproduction. Reprod. Biol. Endocrinol. 3:28. doi: 10.1186/1477-7827-3-28

Agarwal, A., Mulgund, A., Hamada, A., and Chyatte, M. R. (2015). A unique view on male infertility around the globe. Reprod. Biol. Endocrinol. 13:37. doi: 10.1186/s12958-015-0032-1

Aldawood, N., Alrezaki, A., Alanazi, S., Amor, N., Alwasel, S., Sirotkin, A., et al. (2020). Acrylamide impairs ovarian function by promoting apoptosis and affecting reproductive hormone release, steroidogenesis and autophagy-related

\section{DATA AVAILABILITY STATEMENT}

The original contributions generated for this study are included in the article/Supplementary Material, further inquiries can be directed to the corresponding author.

\section{ETHICS STATEMENT}

The animal study was reviewed and approved by University of Newcastle Animal Care \& Ethics Committee.

\section{AUTHOR CONTRIBUTIONS}

AP and JS contributed substantially to conception and design, acquisition of data, analysis and interpretation of data, drafting the article and revising it critically for important intellectual content, and final approval of the version to be published. SC, $\mathrm{SR}, \mathrm{EB}$, and $\mathrm{BN}$ contributed to acquisition of data, analysis and interpretation of data, critical revision of article for important intellectual content, and final approval of the version to be published. EM contributed to critical revision of article for important intellectual content and final approval of the version to be published. All authors contributed to the article and approved the submitted version.

\section{FUNDING}

AP is a recipient of a University of Newcastle Postgraduate Research Scholarship. BN is a recipient of a National Health and Medical Research Council Senior Research Fellowship (APP1154837). JS is a recipient of an Australian National Health and Medical Research Council Early Career Fellowship (GNT1120481) and the Hunter Medical Research Institute Bob and Terry Kennedy Children's Research Project Grant in Pregnancy and Reproduction (G1501433 and G1801335).

\section{SUPPLEMENTARY MATERIAL}

The Supplementary Material for this article can be found online at: https://www.frontiersin.org/articles/10.3389/fcell.2021. 691826/full\#supplementary-material

genes: an in vivo study. Ecotoxicol. Environ. Saf. 197:110595. doi: 10.1016/j. ecoenv.2020.110595

Bajaj, L., Lotfi, P., Pal, R., Ronza, A. D., Sharma, J., and Sardiello, M. (2019). Lysosome biogenesis in health and disease. J. Neurochem. 148, 573-589. doi: $10.1111 /$ jnc. 14564

Bang, S., Shin, H., Song, H., Suh, C. S., and Lim, H. J. (2014). Autophagic activation in vitrified-warmed mouse oocytes. Reproduction 148, 11-19. doi: 10.1530/rep14-0036

Berg, T. O., Fengsrud, M., Strømhaug, P. E., Berg, T., and Seglen, P. O. (1998). Isolation and characterization of rat liver amphisomes: evidence for fusion 
of autophagosomes with both early and late endosomes. J. Biol. Chem. 273, 21883-21892. doi: 10.1074/jbc.273.34.21883

Brunk, U. T., Dalen, H., Roberg, K., and Hellquist, H. B. (1997). Photo-oxidative disruption of lysosomal membranes causes apoptosis of cultured human fibroblasts. Free Radic. Biol. Med. 23, 616-626. doi: 10.1016/s0891-5849(97) 00007-5

Camlin, N. J., Mclaughlin, E. A., and Holt, J. E. (2017a). Motoring through: the role of kinesin superfamily proteins in female meiosis. Hum. Reprod. Update 23, 409-420. doi: 10.1093/humupd/dmx010

Camlin, N. J., Mclaughlin, E., and Holt, J. E. (2017b). The use of C57Bl/6× CBA F1 hybrid cross as a model for human age-related oocyte aneuploidy. Mol Reprod Dev. 84, 6-7. doi: 10.1002/mrd.22766

Chao, H. T., Lee, S. Y., Lee, H. M., Liao, T. L., Wei, Y. H., and Kau, S. H. (2005). Repeated ovarian stimulations induce oxidative damage and mitochondrial DNA mutations in mouse ovaries. Ann. N. Y. Acad. Sci. 1042, 148-156. doi: 10.1196/annals.1338.016

Chaudhary, G. R., Yadav, P. K., Yadav, A. K., Tiwari, M., Gupta, A., Sharma, A., et al. (2019). Necrosis and necroptosis in germ cell depletion from mammalian ovary. J. Cel. Physiol. 234, 8019-8027. doi: 10.1002/jcp.27562

Christians, E., Boiani, M., Garagna, S., Dessy, C., Redi, C. A., Renard, J. P., et al. (1999). Gene expression and chromatin organization during mouse oocyte growth. Dev. Biol. 207, 76-85. doi: 10.1006/dbio.1998.9157

Cortes, C. J., and La Spada, A. R. (2019). TFEB dysregulation as a driver of autophagy dysfunction in neurodegenerative disease: molecular mechanisms, cellular processes, and emerging therapeutic opportunities. Neurobiol. Dis. 122, 83-93. doi: 10.1016/j.nbd.2018.05.012

Cuervo, A. M., Bergamini, E., Brunk, U. T., Dröge, W., Ffrench, M., and Terman, A. (2005). Autophagy and aging: the importance of maintaining "Clean" Cells. Autophagy 1, 131-140. doi: 10.4161/auto.1.3.2017

De Bruin, J., and Te Velde, E. (2004). Female reproductive ageing: concepts and consequences. Preserv. Fertil. 3, 1-19.

Doherty, L., and Pal, L. (2011). Reproductive banking and older women. Maturitas 70, 3-4. doi: 10.1016/j.maturitas.2011.06.012

Escobar, M. L., Echeverría, O. M., Ortíz, R., and Vázquez-Nin, G. H. (2008). Combined apoptosis and autophagy, the process that eliminates the oocytes of atretic follicles in immature rats. Apoptosis 13, 1253-1266. doi: 10.1007/s10495008-0248-z

Essick, E. E., and Sam, F. (2010). Oxidative stress and autophagy in cardiac disease, neurological disorders, aging and cancer. Oxid. Med. Cell. Longev. 3, 168-177. doi: 10.4161/oxim.3.3.12106

Feng, Y., He, D., Yao, Z., and Klionsky, D. J. (2014). The machinery of macroautophagy. Cell. Res. 24, 24-41. doi: 10.1038/cr.2013.168

Fu, X., Cheng, J., Hou, Y., and Zhu, S. (2014). The association between the oocyte pool and aneuploidy: a comparative study of the reproductive potential of young and aged mice. J. Assist. Reprod. Genet. 31, 323-331. doi: 10.1007/ s10815-013-0160-5

Gawriluk, T. R., Hale, A. N., Flaws, J. A., Dillon, C. P., Green, D. R., and Rucker, E. B. (2011). Autophagy is a cell survival program for female germ cells in the murine ovary. Reproduction 141, 759-765. doi: 10.1530/rep-10-0489

Gilchrist, R. B., Lane, M., and Thompson, J. G. (2008). Oocyte-secreted factors: regulators of cumulus cell function and oocyte quality. Hum. Reprod. Update 14, 159-177. doi: 10.1093/humupd/dmm040

Goud, A. P., Goud, P. T., Diamond, M. P., Gonik, B., and Abu-Soud, H. M. (2008). Reactive oxygen species and oocyte aging: role of superoxide, hydrogen peroxide, and hypochlorous acid. Free Radic. Biol. Med. 44, 1295-1304. doi: 10.1016/j.freeradbiomed.2007.11.014

Hajimolaali, M., Mohammadian, H., Torabi, A., Shirini, A., Khalife Shal, M., Barazandeh Nezhad, H., et al. (2021). Application of chloroquine as an endosomal escape enhancing agent: new frontiers for an old drug. Expert Opin. Drug Deliv. 1-13. doi: 10.1080/17425247.2021.1873272 [Epub ahead of print].

Hirokawa, N., Noda, Y., Tanaka, Y., and Niwa, S. (2009). Kinesin superfamily motor proteins and intracellular transport. Nat. Rev. Mol. Cell Biol. 10, 682-696. doi: $10.1038 / \mathrm{nrm} 2774$

Hsueh, A. J., Kawamura, K., Cheng, Y., and Fauser, B. C. (2015). Intraovarian control of early folliculogenesis. Endocr. Rev. 36, 1-24. doi: 10.1210/er.20141020

Jia, B., Xue, Y., Yan, X., Li, J., Wu, Y., Guo, R., et al. (2018). Autophagy inhibitor chloroquine induces apoptosis of cholangiocarcinoma cells via endoplasmic reticulum stress. Oncol. Lett. 16, 3509-3516. doi: 10.3892/ol.2018. 9131

Kamal, A., and Goldstein, L. S. B. (2000). Connecting vesicle transport to the cytoskeleton. Curr. Opin. Cell Biol. 12, 503-508. doi: 10.1016/s0955-0674(00) 00123-x

Kaur, G., and Lakkaraju, A. (2018). Early endosome morphology in health and disease. Adv. Exp. Med. Biol. 1074, 335-343. doi: 10.1007/978-3-319-754024_41

Klionsky, D. J., Abdelmohsen, K., Abe, A., Abedin, M. J., Abeliovich, H., Acevedo Arozena, A., et al. (2016). Guidelines for the use and interpretation of assays for monitoring autophagy. Autophagy 12, 1-222.

Klionsky, D. J., and Codogno, P. (2013). The mechanism and physiological function of macroautophagy. J. Innate Immun. 5, 427-433. doi: 10.1159/ 000351979

Klionsky, D. J., Eskelinen, E.-L., and Deretic, V. (2014). Autophagosomes, phagosomes, autolysosomes, phagolysosomes, autophagolysosomes... Wait. I'm confused. Autophagy 10, 549-551. doi: 10.4161/auto.28448

Koren, I., Reem, E., and Kimchi, A. (2010). Autophagy gets a brake. Autophagy 6, 1179-1180. doi: 10.4161/auto.6.8.13338

Kuliev, A., Cieslak, J., Ilkevitch, Y., and Verlinsky, Y. (2003). Chromosomal abnormalities in a series of 6733 human oocytes in preimplantation diagnosis for age-related aneuploidies. Reprod. Biomed. Online 6, 54-59. doi: 10.1016/ s1472-6483(10)62055-x

Latorraca, L. B., Feitosa, W. B., Mariano, C., Moura, M. T., Fontes, P. K., Nogueira, M. F. G., et al. (2020). Autophagy is a pro-survival adaptive response to heat shock in bovine cumulus-oocyte complexes. Sci. Rep. 10:13711.

Legland, D., Arganda-Carreras, I., and Andrey, P. (2016). MorphoLibJ: integrated library and plugins for mathematical morphology with ImageJ. Bioinformatics 32, 3532-3534.

Leridon, H. (2004). Can assisted reproduction technology compensate for the natural decline in fertility with age? A model assessment. Hum. Reprod. 19, 1548-1553. doi: 10.1093/humrep/deh304

Levine, B., and Kroemer, G. (2008). Autophagy in the pathogenesis of disease. Cell 132, 27-42. doi: 10.1016/j.cell.2007.12.018

Lliberos, C., Liew, S. H., Zareie, P., La Gruta, N. L., Mansell, A., and Hutt, K. (2021). Evaluation of inflammation and follicle depletion during ovarian ageing in mice. Sci. Rep. 11:278.

Luzio, J. P., Pryor, P. R., and Bright, N. A. (2007). Lysosomes: fusion and function. Nat. Rev. Mol. Cell Biol. 8, 622-632. doi: 10.1038/nrm2217

Martinet, W., Schrijvers, D. M., Timmermans, J.-P., Bult, H., and De Meyer, G. R. Y. (2013). Immunohistochemical analysis of macroautophagy. Autophagy 9, 386-402. doi: 10.4161/auto.22968

Mauthe, M., Orhon, I., Rocchi, C., Zhou, X., Luhr, M., Hijlkema, K.-J., et al. (2018). Chloroquine inhibits autophagic flux by decreasing autophagosome-lysosome fusion. Autophagy 14, 1435-1455. doi: 10.1080/15548627.2018.1474314

Miao, J.-K., Liu, Y.-H., Liu, S., Liu, X.-M., Wang, P.-C., Du, Z.-Q., et al. (2019). Lysosomal dysfunction disturbs porcine oocyte maturation and developmental capacity by disorganizing chromosome/cytoskeleton and activating autophagy/apoptosis. Theriogenology 140, 44-51. doi: 10.1016/j. theriogenology.2019.08.019

Mihalas, B. P., Bromfield, E. G., Sutherland, J. M., De Iuliis, G. N., Mclaughlin, E. A., Aitken, R. J., et al. (2018). Oxidative damage in naturally aged mouse oocytes is exacerbated by dysregulation of proteasomal activity. J. Biol. Chem. 293, 18944-18964. doi: 10.1074/jbc.ra118.005751

Mihalas, B. P., Camlin, N. J., Xavier, M. J., Peters, A. E., Holt, J. E., Sutherland, J. M., et al. (2019). The small non-coding RNA profile of mouse oocytes is modified during aging. Aging 11, 2968-2997. doi: 10.18632/aging.101947

Miyamoto, K., Sato, E. F., Kasahara, E., Jikumaru, M., Hiramoto, K., Tabata, H., et al. (2010). Effect of oxidative stress during repeated ovulation on the structure and functions of the ovary, oocytes, and their mitochondria. Free Radic. Biol. Med. 49, 674-681. doi: 10.1016/j.freeradbiomed.2010.05.025

Mizushima, N., Ohsumi, Y., and Yoshimori, T. (2002). Autophagosome formation in mammalian cells. Cell Struct. Funct. 27, 421-429. doi: 10.1247/csf.27.421

Muralidharan, H., and Baas, P. W. (2019). Mitotic Motor KIFC1 is an organizer of microtubules in the axon. J. Neurosci. 39, 3792-3811. doi: 10.1523/jneurosci. 3099-18.2019

Myung Park, J., Tougeron, D., Huang, S., Okamoto, K., and Sinicrope, F. A. (2014). Beclin 1 and UVRAG confer protection from radiation-induced DNA 
damage and maintain centrosome stability in colorectal cancer cells. PLoS One 9:e100819. doi: 10.1371/journal.pone.0100819

Nakamura, S., and Yoshimori, T. (2017). New insights into autophagosomelysosome fusion. J. Cell. Sci. 130, 1209-1216.

Newman, J. E., Fitzgerald, O., Paul, R. C., and Chambers, G. M. (2019). Assisted Reproductive Technology in Australia and New Zealand 2017. Sydney, NSW: University of New South Wales Sydney.

Pellegrini, P., Strambi, A., Zipoli, C., Hägg-Olofsson, M., Buoncervello, M., Linder, S., et al. (2014). Acidic extracellular $\mathrm{pH}$ neutralizes the autophagy-inhibiting activity of chloroquine. Autophagy 10, 562-571. doi: 10.4161/auto.27901

Peters, A. E., Mihalas, B. P., Bromfield, E. G., Roman, S. D., Nixon, B., and Sutherland, J. M. (2020). Autophagy in female fertility: a role in oxidative stress and aging. Antioxid. Redox. Signal. 32, 550-568. doi: 10.1089/ars.2019.7986

Ponsford, A. H., Ryan, T. A., Raimondi, A., Cocucci, E., Wycislo, S. A., Fröhlich, F., et al. (2020). Live imaging of intra-lysosome $\mathrm{pH}$ in cell lines and primary neuronal culture using a novel genetically encoded biosensor. Autophagy 17, $1-19$.

Qvarnström, O. F., Simonsson, M., Johansson, K.-A., Nyman, J., and Turesson, I. (2004). DNA double strand break quantification in skin biopsies. Radiother. Oncol. 72, 311-317. doi: 10.1016/j.radonc.2004.07.009

Saftig, P., and Klumperman, J. (2009). Lysosome biogenesis and lysosomal membrane proteins: trafficking meets function. Nat. Rev. Mol. Cell Biol. 10, 623-635. doi: 10.1038/nrm 2745

Sardiello, M. (2016). Transcription factor EB: from master coordinator of lysosomal pathways to candidate therapeutic target in degenerative storage diseases. Ann. N. Y. Acad. Sci. 1371, 3-14. doi: 10.1111/nyas.13131

Schindelin, J., Arganda-Carreras, I., Frise, E., Kaynig, V., Longair, M., Pietzsch, T., et al. (2012). Fiji: an open-source platform for biological-image analysis. Nat. Methods 9, 676-682. doi: 10.1038/nmeth.2019

Schmittgen, T. D., and Livak, K. J. (2008). Analyzing real-time PCR data by the comparative C T method. Nat. Protoc. 3, 1101-1108. doi: 10.1038/nprot. 2008.73

Seglen, P. O., Gordon, P., and Holen, I. (1990). Non-selective autophagy. Semin. Cell. Biol. 1, 441-448.

Shen, X.-H., Jin, Y.-X., Liang, S., Kwon, J.-W., Zhu, J.-W., Lei, L., et al. (2018). Autophagy is required for proper meiosis of porcine oocytes maturing in vitro. Sci. Rep. 8:12581.

Skjeldal, F. M., Strunze, S., Bergeland, T., Walseng, E., Gregers, T. F., and Bakke, O. (2012). The fusion of early endosomes induces molecular-motor-driven tubule formation and fission. J. Cell. Sci. 125, 1910-1919.

Spira, A. (1988). The decline of fecundity with age. Maturitas 10, 15-22. doi: 10.1016/0378-5122(88)90004-7

Tammineni, P., and Cai, Q. (2017). Defective retrograde transport impairs autophagic clearance in Alzheimer disease neurons. Autophagy 13, 982-984. doi: 10.1080/15548627.2017.1291114

te Velde, E. R., and Pearson, P. L. (2002). The variability of female reproductive ageing. Hum. Reprod. Update 8, 141-154. doi: 10.1093/humupd/8.2.141

Terman, A. (1995). The effect of age on formation and elimination of autophagic vacuoles in mouse hepatocytes. Gerontology 41, 319-326. doi: 10.1159/ 000213753

Terman, A., and Brunk, U. T. (2004). Lipofuscin. Int. J. Biochem. Cell. Biol. 36, 1400-1404.
Titus, S., Li, F., Stobezki, R., Akula, K., Unsal, E., Jeong, K., et al. (2013). Impairment of BRCA1-related DNA double-strand break repair leads to ovarian aging in mice and humans. Sci. Transl. Med. 5:172ra121.

Trivedi, P. C., Bartlett, J. J., and Pulinilkunnil, T. (2020). Lysosomal biology and function: modern view of cellular debris bin. Cells 9:1131. doi: 10.3390/ cells 9051131

Turan, V., and Oktay, K. (2020). BRCA-related ATM-mediated DNA doublestrand break repair and ovarian aging. Hum. Reprod. Update 26, 43-57. doi: 10.1093/humupd/dmz043

Vander Borght, M., and Wyns, C. (2018). Fertility and infertility: definition and epidemiology. Clin. Biochem. 62, 2-10. doi: 10.1016/j.clinbiochem.2018.03. 012

Vayena, E., Rowe, P. J., and Griffin, P. D. (2002). Current Practices and Controversies in Assisted Reproduction: Report of a Meeting on Medical, Ethical and Social Aspects of Assisted Reproduction, Held at WHO Headquarters in Geneva, Switzerland. Geneva: World Health Organization.

Vilchez, D., Saez, I., and Dillin, A. (2014). The role of protein clearance mechanisms in organismal ageing and age-related diseases. Nat. Commun. 5:5659.

Villari, G., Enrico Bena, C., Del Giudice, M., Gioelli, N., Sandri, C., Camillo, C., et al. (2020). Distinct retrograde microtubule motor sets drive early and late endosome transport. EMBO J. 39:e103661.

Wegner, C. S., Malerød, L., Pedersen, N. M., Progida, C., Bakke, O., Stenmark, H., et al. (2010). Ultrastructural characterization of giant endosomes induced by GTPase-deficient Rab5. Histochem. Cell. Biol. 133, 41-55. doi: 10.1007/s00418009-0643-8

Wilhelm, T., and Richly, H. (2018). Autophagy during ageing - from Dr Jekyll to Mr Hyde. FEBS J. 285, 2367-2376. doi: 10.1111/febs.14453

Wu, L. L., Russell, D. L., Wong, S. L., Chen, M., Tsai, T.-S., St John, J. C., et al. (2015). Mitochondrial dysfunction in oocytes of obese mothers: transmission to offspring and reversal by pharmacological endoplasmic reticulum stress inhibitors. Development 142, 681-691. doi: 10.1242/dev.114850

Xu, F., Fang, Y., Yan, L., Xu, L., Zhang, S., Cao, Y., et al. (2017). Nuclear localization of Beclin 1 promotes radiation-induced DNA damage repair independent of autophagy. Sci. Rep. 7:45385.

Yadav, P. K., Tiwari, M., Gupta, A., Sharma, A., Prasad, S., Pandey, A. N., et al. (2018). Germ cell depletion from mammalian ovary: possible involvement of apoptosis and autophagy. J.Biomed. Sci. 25:36.

Yu, F., Li, J., Xie, Y., Sleightholm, R. L., and Oupický, D. (2016). Polymeric chloroquine as an inhibitor of cancer cell migration and experimental lung metastasis. J. Control. Release 244, 347-356. doi: 10.1016/j.jconrel.2016.07.040

Conflict of Interest: The authors declare that the research was conducted in the absence of any commercial or financial relationships that could be construed as a potential conflict of interest.

Copyright (c) 2021 Peters, Caban, McLaughlin, Roman, Bromfield, Nixon and Sutherland. This is an open-access article distributed under the terms of the Creative Commons Attribution License (CC BY). The use, distribution or reproduction in other forums is permitted, provided the original author(s) and the copyright owner(s) are credited and that the original publication in this journal is cited, in accordance with accepted academic practice. No use, distribution or reproduction is permitted which does not comply with these terms. 